\title{
Impacts of triglyceride-glucose index on prognosis of patients with type 2 diabetes mellitus and non-ST-segment elevation acute coronary syndrome: results from an observational cohort study in China
}

\author{
Qi Zhao' ${ }^{1}$, Ting-Yu Zhang ${ }^{1}$, Yu-Jing Cheng ${ }^{1}$, Yue Ma ${ }^{2}$, Ying-Kai Xu' ${ }^{1}$, Jia-Qi Yang ${ }^{1}$ and Yu-Jie Zhou ${ }^{1 *}$ (D)
}

\begin{abstract}
Background: The relationship between triglyceride-glucose index (TyG index) and the prevalence and prognosis of cardiovascular disease has been confirmed by former studies. However, it remains uncertain whether TyG index has a prognostic impact in patients with type 2 diabetes mellitus (T2DM) and non-ST-segment elevation acute coronary syndrome (NSTE-ACS) undergoing percutaneous coronary intervention (PCI).

Methods: The study retrospectively enrolled 798 patients (mean age: $60.9 \pm 8.3$ years; $68.3 \%$ men) with T2DM and NSTE-ACS who underwent PCl at Beijing Anzhen Hospital from January to December 2015. TyG index was calculated as previously reported: In [fasting TGs (mg/dL) * FBG (mg/dL)/2]. The primary endpoint was a composite of adverse events as follows: all-cause death, non-fatal myocardial infarction (MI) and ischemia-driven revascularization.

Results: TyG index was significantly higher in patients with a primary endpoint event compared with those without. Multivariate Cox proportional hazards analysis showed that 1-unit increase of TyG index was independently associated with higher risk of primary endpoint, independent of other risk factors [hazard ratio (HR) 3.208 per 1-unit increase, 95\% confidence interval (CI) 2.400-4.289, P<0.001]. The addition of TyG index to a baseline risk model had an incremental effect on the predictive value for adverse prognosis [AUC: baseline risk model, $0.800 \mathrm{vs}$. baseline risk model + TyG index, 0.856 , P for comparison $<0.001$; category-free net reclassification improvement (NRI) 0.346 , $\mathrm{P}<0.001$; integrated discrimination improvement (IDI) $0.087, \mathrm{P}<0.001]$.
\end{abstract}

Conclusions: Increased TyG index is a significant predictor of adverse prognosis in patients with T2DM and NSTE-ACS undergoing $\mathrm{PCl}$. Further studies need to be performed to determine whether interventions for TyG index have a positive impact on improving clinical prognosis.

Keywords: Triglyceride-glucose index, Type 2 diabetes mellitus, Non-ST-segment elevation acute coronary syndrome, Percutaneous coronary intervention

\footnotetext{
*Correspondence: azzyj12@163.com

1 Department of Cardiology, Beijing Anzhen Hospital, Capital Medical University, Beijing Institute of Heart Lung and Blood Vessel Disease, Beijing Key Laboratory of Precision Medicine of Coronary Atherosclerotic Disease, Clinical Center for Coronary Heart Disease, Capital Medical University, Beijing 100029, China

Full list of author information is available at the end of the article
}

\section{Background}

Coronary artery disease (CAD) has been recognized as the leading cause of disability and mortality in contemporary society. In recent years, in spite of superior evidence-based strategies including optimized drug therapy

(c) The Author(s) 2020. This article is licensed under a Creative Commons Attribution 4.0 International License, which permits use, sharing, adaptation, distribution and reproduction in any medium or format, as long as you give appropriate credit to the original author(s) and the source, provide a link to the Creative Commons licence, and indicate if changes were made. The images or other third party material in this article are included in the article's Creative Commons licence, unless indicated otherwise in a credit line to the material. If material is not included in the article's Creative Commons licence and your intended use is not permitted by statutory regulation or exceeds the permitted use, you will need to obtain permission directly from the copyright holder. To view a copy of this licence, visit http://creativeco mmons.org/licenses/by/4.0/. The Creative Commons Public Domain Dedication waiver (http://creativecommons.org/publicdomain/ zero/1.0/) applies to the data made available in this article, unless otherwise stated in a credit line to the data. 
and revascularization having been widely developed and applied, the risk of recurrent adverse cardiovascular outcomes remains relatively high in patients with CAD, especially for those who have ever had an acute coronary syndrome (ACS) [1-3]. Previous studies have suggested that more than one-quarter of patients with ACS are combined with Type 2 Diabetes mellitus (T2DM), which has been widely proved to be one of the most significant risk factors for cardiovascular disease [4]. Certain studies have demonstrated that T2DM is significantly correlated with higher prevalence of CAD, more complex coronary lesions and worse prognosis [4-6]. Therefore, identification of the residual risk factors of diabetic patients with ACS is of great clinical importance if we are to develop new therapeutic targets and to tailor risk reduction strategies that match individual risk level.

Insulin resistance (IR), the critical mechanism of the pathogenesis of T2DM, has been extensively demonstrated to be significantly related to the development of coronary and carotid atherosclerosis and an increased risk of adverse prognosis [7-10]. The triglyceride-glucose index (TyG index), which is derived from fasting triglycerides (TGs) and fasting blood glucose (FBG), has been proposed as a surrogate biomarker of IR and former studies have proved that it has high correlation with hyperinsulinaemic-euglycaemic clamp (the gold standard technique for assessing IR), either in individuals with or without T2DM [11-13]. Studies have shown that an increased level of TyG index is closely related to higher incidence of diabetes and prediabetic status [14-16]. Furthermore, the association between TyG index and the prevalence and prognosis of cardiovascular disease has been confirmed by certain clinical researches, despite the existence of diabetes or not at baseline [17-22].

However, the prognostic significance of TyG index in patients with T2DM and non-ST-segment elevation acute coronary syndrome (NSTE-ACS) who were treated with percutaneous coronary intervention (PCI) has not been fully studied. Based on this, the present study was designed with the aim of: (1) identifying the potential association between IR quantified by TyG index and clinical prognosis; (2) determining whether TyG index has an incremental effect on risk stratification on the basis of traditional risk factors in participants with T2DM and NSTE-ACS undergoing PCI.

\section{Methods}

\section{Study population}

The present study is a single-center, observational, retrospective cohort study among patients with diabetes who were diagnosed with NSTE-ACS and treated with elective PCI at Beijing Anzhen Hospital between January and December 2015. The exclusion criteria were listed as follows: (1) explicit or suspected type 1 diabetes mellitus (T1DM); (2) missing clinical data; (3) history of coronary artery bypass grafting (CABG), cardiogenic shock, chronic infectious disease and malignant tumor; (4) taking TGs-lowering medications before admission (such as fibrates); (5) extreme body mass index (BMI) $\left(\mathrm{BMI}>45 \mathrm{~kg} / \mathrm{m}^{2}\right)$ and suspected familial hypertriglyceridemia [plasma TGs $\geq 500 \mathrm{mg} / \mathrm{dL}(5.65 \mathrm{mmol} / \mathrm{L})$ ]; (6) renal dysfunction with estimated glomerular filtration rate $(\mathrm{eGFR})<30 \mathrm{~mL} /\left(\mathrm{min}^{*} 1.73 \mathrm{~m}^{2}\right)$ or treated with renal replacement therapy, severe hepatic insufficiency with alanine transaminase (ALT) or aspartate transaminase (AST) $\geq 5$ upper limit of normal; (7) PCI failure, PCIrelated complications, and in-hospital death. Ultimately, a cohort of 798 patients who met the enrollment principles were included for the present analyses (Fig. 1).

\section{Data collection and definitions}

Data of demographic and clinical characteristics, including age, sex, weight, height, heart rate, blood pressure [systolic blood pressure (SBP) and diastolic blood pressure (DBP)], medical history, family history, and medical treatment were extracted from the medical information recording system of Beijing Anzhen Hospital. BMI was calculated as follows: $\mathrm{BMI}=$ weight $(\mathrm{kg}) /[\text { height }(\mathrm{m})]^{2}$. Criteria for diabetes include: (1) previously diagnosed diabetes under treatment of antidiabetic medication (diet, oral agents, and/or insulin); (2) the typical symptoms of diabetes with a random blood glucose $\geq 11.1 \mathrm{mmol} / \mathrm{L}$, and/or FBG $\geq 7.0 \mathrm{mmol} / \mathrm{L}$, and/or 2-h blood glucose after oral glucose tolerance test $(\mathrm{OGTT}) \geq 11.1 \mathrm{mmol} / \mathrm{L}$ [23]; (3) glycosylated hemoglobin A1c (HbA1c) level $\geq 6.5 \%$ on admission [24]. NSTE-ACS was composed of nonST-segment elevation myocardial infarction (NSTEMI) and unstable angina (UA), definitions of which were determined by appropriate guidelines [25]. NSTEMI was defined as having symptoms of ischemia and elevated cardiac troponin I (cTnI), and without an elevation of ST-segment. UA was diagnosed as ischemic symptoms at rest, or exacerbated or new-onset symptoms with transient ischemic ST-segment shifts, and without release of myocardial enzymes related to myocardial necrosis. Patients with SBP $\geq 140 \mathrm{mmHg}$ and/or DBP $\geq 90 \mathrm{mmHg}$, or those receiving anti-hypertensive treatments were considered having hypertension. Peripheral vascular disease (PVD) was defined as aorta and other arteries than coronary arteries, with exercise related claudication, or reduced or absent pulsation, or angiographic stenosis of more than $50 \%$.

Venous blood samples were collected after an overnight fasting on the day of the baseline coronary procedure. The routine hematology and biochemical parameters, including lipid profiles [TGs, total 
cholesterol (TC), low-density lipoprotein cholesterol (LDL-C), high-density lipoprotein cholesterol (HDLC)], creatinine, uric acid, FBG, HbA1c, high-sensitivity C-reactive protein (hs-CRP), and other biomarkers, were determined by standard laboratory methods in central lab of Beijing Anzhen Hospital. Patients with fasting TC $>200 \mathrm{mg} / \mathrm{dL}$, and/or LDL-C $>130 \mathrm{mg} / \mathrm{dL}$, and/or TGs $>150 \mathrm{mg} / \mathrm{dL}$, and/or HDL-C $<40 \mathrm{mg} / \mathrm{dL}$ at admission, and/or previously long-term use of lipidlowering drugs were considered having dyslipidemia. The eGFR was calculated as previously described: eGFR $\left[\mathrm{mL} /\left(\min * 1.73 \mathrm{~m}^{2}\right)\right]=186 *$ serum creatinine

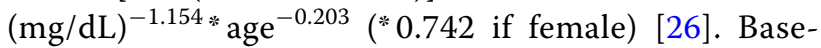
line TyG index was calculated based on fasting TGs and FBG values obtained at admission as previously reported: ln [fasting TGs $(\mathrm{mg} / \mathrm{dL}){ }^{*} \mathrm{FBG}(\mathrm{mg} / \mathrm{dL}) / 2$ ] [11]. Left ventricular ejection fraction (LVEF) was evaluated by two-dimensional modified Simpson's method using an ultrasonic cardiogram (Philips Company, Eindhoven, The Netherlands).

Coronary angiogram data were analyzed and recorded by at least two experienced cardiologists, and measurements of coronary artery lesion characteristics were obtained. The lesion characteristics were defined as follows: (1) multi-vessel lesion: more than two main coronary branches (vessel diameter $\geq 2 \mathrm{~mm}$ ) with extent of stenosis $\geq 50 \%$. (2) chronic total occlusion lesion: lesion with complete obstruction [thrombolysis in myocardial infarction (TIMI) flow grade 0] lasting longer than 3 months, which was judged from the previous medical history or coronary angiogram results. (3) diffuse lesion: a single stenotic lesion with a length of $\geq 20 \mathrm{~mm}$. (4) bifurcation lesion: stenosis occurred adjacent to and/or involving the origin of a significant side branch that has too much functional value and so cannot be lost during the interventional procedure. (5) in-stent restenosis: stenosis of $\geq 50 \%$ occurring in the segment inside the stent, $5 \mathrm{~mm}$ proximal or distal to the stent [27]. The severity of coronary artery lesions was quantified by the synergy between PCI with taxus and cardiac surgery (SYNTAX) score. The SYNTAX score was calculated for each participant using the online calculator (http://www.synta xscore.com). PCI was performed in accordance with current practice guidelines in China [28], and detailed strategies were determined by experienced interventional cardiologists.

\section{Follow-up and endpoint event}

After baseline PCI, all patients were routinely followed up by trained professionals who were blinded to the baseline information at 3, 6, and 12 months and then annually for up to 36 months. The information about adverse prognostic events was obtained from patients or their family members by telephone questionnaire. The information was further confirmed by careful verification of corresponding medical records if necessary. The primary observational endpoint was defined as a composite of events including all-cause death, non-fatal myocardial infarction (MI) and ischemia-driven revascularization. The secondary observational endpoints were each component of the composite primary endpoint. MI was defined as elevated cardiac troponin higher than the upper reference limit with ischemia indicated from symptoms and/or electrocardiographic changes, with or without an elevation of ST-segment. Ischemia-driven revascularization was defined as the revascularization procedure associated with symptoms and/or electrocardiographic changes implicating ischemia. The first primary endpoint event that occurred during the follow-up was used for analysis in current study. For patients with multiple adverse outcomes occurring almost simultaneously during the follow-up, only the most severe event (all-cause death $>$ non-fatal $\mathrm{MI}>$ ischemia-driven revascularization) was selected to perform our analyses. If the same event occurs multiple times, only the first occurrence was used for analysis.

\section{Statistical analysis}

Continuous variables were presented as mean \pm standard deviation (SD) or median (25th and 75th percentiles: P25, P75) in the case of normal or non-normal distribution, and differences between the two groups were examined by independent-sample t-test or Mann-Whitney $U$ test correspondingly. Categorical variables were described as counts (percentages) and compared by Pearson chisquare test (Pearson $\chi^{2}$ test) or Fisher's exact test appropriately. The Spearman's rank correlation test or Pearson correlation test was used for evaluating the correlations between the TyG index and cardiovascular risk factors when appropriate. The Pearson correlation test was used to evaluate the correlation between two continuous variables with normal distribution, while the Spearman's rank correlation test was applied in case that one or more of the variables being analyzed was non-normally distributed continuous variable or categorical variable. Receiver-operating characteristic (ROC) curve analysis was performed to determine the optimal cutoff point value of TyG index for predicting primary endpoint. The Kaplan-Meier survival analyses were performed to evaluate the incidence rate of adverse events between groups according to the optimal cutoff point of TyG index, and discrepancies between groups were evaluated by log-rank test. The predictive value of the variables for primary endpoint was evaluated by univariate 
and multivariate Cox proportional hazards analyses. The TyG index was analyzed in two ways: (1) as a categorical variable; and (2) as a continuous variable. In multivariate Cox proportional hazards analyses, four models were established to evaluate the predictive value of TyG index for primary endpoint, among which confounders were selected according to statistical significance $(\mathrm{P}<0.2)$ in univariate analysis and clinical importance: (1) Model 1: adjusted for age, sex (female), BMI, SBP, DBP, smoking, drinking, duration of diabetes, dyslipidemia, prior MI, PCI, stroke and PVD; (2) Model 2: adjusted for variables included in Model 1 and diagnosis (NSTEMI), TC, HDL-C, eGFR, HbA1c, LVEF; (3) Model 3: adjusted for variables included in Model 2 and SYNTAX score, left main artery (LM) treatment, drug-coated balloon (DCB) use, complete revascularization and number of stents; (4) Model 4: adjusted for variables included in Model 3 and dual antiplatelet therapy (DAPT) at discharge, DAPT interruption in 12 months, statins at discharge, statins interruption in 12 months, oral hypoglycemic agents (metformin, alpha-glucosidase inhibitor, sulfonylurea, dipeptidyl peptidase 4 inhibitor) at discharge and insulin at discharge. The prognostic impact of TyG index for each component of primary endpoint was also assessed by using model 4 . FBG and TGs were not introduced into multivariate analysis since the TyG index was calculated from them. Results of Cox proportional hazards analyses were presented as hazard ratio (HR) and 95\% confidence intervals $(\mathrm{CI})$. Further stratified analyses according to age ( $\leq 65$ and $>65$ years), sex, BMI ( $\leq 28$ and $>28 \mathrm{~kg}$ / $\mathrm{m}^{2}$ ), hypertension, initial diagnosis (UA and NSTEMI), HbA1c ( $\leq 7$ and $>7 \%)$, LDL-C $(\leq 70$ and $>70 \mathrm{mg} / \mathrm{dL})$, and pre-admission medication including statins, oral hypoglycemic agents and insulin were employed to examine the consistence of the prognostic impact of TyG index for primary endpoint. The model used in the stratified analyses consisted of all covariates used in Model 4 except for the variables that were used for stratification. The interaction of TyG index and variables used for stratification was examined by likelihood ratio tests.

C-statistics including ROC curve analysis were performed to examine the incremental effects of TyG index on the predictive potential of the baseline risk model that including traditional risk factors. DeLong's test was used to compare the area under the curve (AUC) from each of the models. We also calculated category-free net reclassification improvement (NRI) and integrated discrimination improvement (IDI) to determine the extent to which the addition of TyG index improves the predictive power of existing baseline risk model.

Statistical tests were performed with SPSS 23.0 (SPSS Inc., Chicago, Illinois, USA), the R Programming Language (version 3.5.1) and MedCalc version 19.1 (MedCalc
Software, Belgium). A two-tailed $\mathrm{P}$ value $<0.05$ was regarded as statistically significant.

\section{Results}

A total of 798 patients (mean age: $60.9 \pm 8.3$ years; $68.3 \%$ men) were finally enrolled in present study. During the 36-month follow-up period, 17 patients $(2.1 \%$ of total population) were lost to follow-up. Among the 798 participants, $180(22.6 \%)$ experienced primary endpoint events, which consisted of $14(1.8 \%)$ all-cause death, 37 (4.6\%) non-fatal MI, and 129 (16.2\%) ischemia-driven revascularization.

\section{Baseline characteristic of study population}

Baseline characteristics of the total population and groups stratified by the occurrence of primary endpoint event were presented in Table 1 . TyG index was significantly higher in patients with primary endpoint event compared with those without. Patients with a primary endpoint event showed higher age and SBP, longer duration of diabetes, and higher prevalence of dyslipidemia, previous MI and PCI history. In terms of laboratory indicators, participants with endpoint event had higher levels of TGs, TC, hs-CRP, FBG and HbA1c, but lower levels of HDL-C, eGFR and LVEF. As for the angiographic findings, those with an endpoint event showed higher proportions of LM disease, multi-vessel disease and other characteristics of complex coronary artery lesion. The SYNTAX score was significantly higher in subset with adverse prognosis. Correspondingly, more LM lesions were disposed and more coronary artery stents were implanted in patients with endpoint event. Moreover, the rate of complete revascularization was significantly lower in participants with adverse prognosis.

ROC curve analysis showed that the AUC of TyG index for predicting primary endpoint was 0.745 (95\% CI $0.702-0.788, \mathrm{P}<0.001)$. The TyG index of 9.18 was determined as the optimal cutoff point for predicting primary endpoint with a sensitivity of $77.2 \%$ and a specificity of $62.8 \%$. Baseline characteristics of groups according to the optimal cutoff point of TyG index were summarized in Table 2. Compared with patients in lower TyG index group, those with higher TyG index seemed to be younger, manifest higher levels of BMI and heart rate, and higher proportion of dyslipidemia. Laboratory indexes including TGs, TC, LDL-C, hs-CRP, uric acid, FBG and HbAlc were significantly higher in patients with higher TyG index, while HDL-C levels were relatively lower. In higher TyG index group, more patients were diagnosed as NSTEMI and prescribed insulin for treatment. Participants with higher TyG index also showed higher SYNTAX score compared to those with lower TyG index. 
Table 1 Baseline clinical characteristics of patients with and without adverse event

\begin{tabular}{|c|c|c|c|c|}
\hline & Total population $(n=798)$ & Without event $(n=618)$ & With event $(n=180)$ & $P$ value \\
\hline Age, years & $60.9 \pm 8.3$ & $60.3 \pm 8.1$ & $62.9 \pm 8.6$ & $<0.001$ \\
\hline Sex, male, n (\%) & $545(68.3)$ & $430(69.6)$ & $115(63.9)$ & 0.149 \\
\hline $\mathrm{BMI}, \mathrm{kg} / \mathrm{m}^{2}$ & $26.7 \pm 3.2$ & $26.7 \pm 3.2$ & $26.6 \pm 3.2$ & 0.772 \\
\hline Heart rate, bpm & $71.7 \pm 10.2$ & $71.5 \pm 9.8$ & $72.1 \pm 11.4$ & 0.502 \\
\hline $\mathrm{SBP}, \mathrm{mmHg}$ & $131.8 \pm 17.1$ & $130.8 \pm 16.3$ & $135.1 \pm 19.5$ & 0.007 \\
\hline $\mathrm{DBP}, \mathrm{mmHg}$ & $76.8 \pm 10.1$ & $76.4 \pm 9.8$ & $78.1 \pm 11.1$ & 0.052 \\
\hline Smoking, n (\%) & $417(52.3)$ & $332(53.7)$ & $85(47.2)$ & 0.124 \\
\hline Drinking, n (\%) & $184(23.1)$ & $149(24.1)$ & $35(19.4)$ & 0.191 \\
\hline Family history of CAD, n (\%) & $93(11.7)$ & $73(11.8)$ & $20(11.1)$ & 0.796 \\
\hline Duration of diabetes, years & $8.2 \pm 4.3$ & $8.0 \pm 4.1$ & $9.1 \pm 4.7$ & 0.007 \\
\hline \multicolumn{5}{|l|}{ Medical history, n (\%) } \\
\hline Hypertension & $573(71.8)$ & $449(72.7)$ & $124(68.9)$ & 0.323 \\
\hline Dyslipidemia & $710(89.0)$ & $537(86.9)$ & $173(96.1)$ & 0.001 \\
\hline Prior Ml & $175(21.9)$ & $118(19.1)$ & $57(31.7)$ & $<0.001$ \\
\hline Prior PCl & $151(18.9)$ & $106(17.2)$ & $45(25.0)$ & 0.018 \\
\hline Prior stroke & $109(13.7)$ & $80(12.9)$ & $29(16.1)$ & 0.276 \\
\hline Prior PVD & $125(15.7)$ & $93(15.0)$ & $32(17.8)$ & 0.375 \\
\hline \multicolumn{5}{|l|}{ Laboratory results } \\
\hline $\mathrm{TGs}, \mathrm{mg} / \mathrm{dL}$ & $138.2(97.2,198.5)$ & $127.6(91.3,174.8)$ & $209.1(134.9,299.5)$ & $<0.001$ \\
\hline $\mathrm{TC}, \mathrm{mg} / \mathrm{dL}$ & $157.2 \pm 39.7$ & $153.5 \pm 39.4$ & $170.2 \pm 38.2$ & $<0.001$ \\
\hline $\mathrm{LDL}-\mathrm{C}, \mathrm{mg} / \mathrm{dL}$ & $94.2 \pm 33.1$ & $93.2 \pm 33.9$ & $97.6 \pm 29.8$ & 0.112 \\
\hline $\mathrm{HDL}-\mathrm{C}, \mathrm{mg} / \mathrm{dL}$ & $36.9 \pm 8.7$ & $37.3 \pm 8.8$ & $35.3 \pm 8.0$ & 0.005 \\
\hline hs-CRP, mg/L & $1.6(0.7,4.1)$ & $1.5(0.6,3.9)$ & $2.0(0.9,4.4)$ & 0.011 \\
\hline Creatinine, mg/dL & $0.8 \pm 0.2$ & $0.8 \pm 0.2$ & $0.8 \pm 0.2$ & 0.384 \\
\hline $\mathrm{eGFR}, \mathrm{mL} /\left(\mathrm{min}^{*} 1.73 \mathrm{~m}^{2}\right)$ & $96.5 \pm 21.6$ & $97.4 \pm 21.7$ & $93.5 \pm 21.1$ & 0.030 \\
\hline Uric acid, $\mu \mathrm{mol} / \mathrm{L}$ & $328.0 \pm 75.6$ & $328.3 \pm 75.3$ & $327.0 \pm 76.6$ & 0.842 \\
\hline $\mathrm{FBG}, \mathrm{mg} / \mathrm{dL}$ & $127.7(109.6,157.0)$ & $125.3(108.1,148.4)$ & $141.5(118.4,173.3)$ & $<0.001$ \\
\hline $\mathrm{HbA} 1 \mathrm{c}, \%$ & $7.5 \pm 1.3$ & $7.3 \pm 1.2$ & $8.0 \pm 1.3$ & $<0.001$ \\
\hline TyG index & $9.1 \pm 0.6$ & $9.0 \pm 0.6$ & $9.6 \pm 0.7$ & $<0.001$ \\
\hline LVEF, \% & $64.0 \pm 6.6$ & $64.3 \pm 6.3$ & $62.7 \pm 7.5$ & 0.010 \\
\hline Initial diagnosis, n (\%) & & & & 0.149 \\
\hline UA & $650(81.5)$ & $510(82.5)$ & $140(77.8)$ & \\
\hline NSTEMI & $148(18.5)$ & $108(17.5)$ & $40(22.2)$ & \\
\hline \multicolumn{5}{|l|}{ Pre-admission medication, n (\%) } \\
\hline ACEl & $79(9.9)$ & $62(10.0)$ & $17(9.4)$ & 0.816 \\
\hline ARB & $128(16.0)$ & $99(16.0)$ & $29(16.1)$ & 0.976 \\
\hline DAPT & $253(31.7)$ & $194(31.4)$ & $59(32.8)$ & 0.725 \\
\hline Aspirin & $427(53.5)$ & $325(52.6)$ & $102(56.7)$ & 0.334 \\
\hline Clopidogrel & $264(33.1)$ & $203(32.8)$ & $61(33.9)$ & 0.794 \\
\hline$\beta$-blocker & $166(20.8)$ & $127(20.6)$ & $39(21.7)$ & 0.745 \\
\hline Statins & $233(29.2)$ & $190(30.7)$ & $43(23.9)$ & 0.075 \\
\hline Proton pump inhibitor & $8(1.0)$ & $7(1.1)$ & $1(0.6)$ & 0.796 \\
\hline Oral hypoglycemic agents & $413(51.8)$ & $324(52.4)$ & $89(49.4)$ & 0.481 \\
\hline Metformin & $170(21.3)$ & $138(22.3)$ & $32(17.8)$ & 0.189 \\
\hline Alpha-glucosidase inhibitor & $185(23.2)$ & $140(22.7)$ & $45(25.0)$ & 0.512 \\
\hline Sulfonylurea & $126(15.8)$ & $102(16.5)$ & $24(13.3)$ & 0.304 \\
\hline Dipeptidyl peptidase 4 inhibitor & $15(1.9)$ & $12(1.9)$ & $3(1.7)$ & 0.811 \\
\hline Insulin & $225(28.2)$ & $163(26.4)$ & $62(34.4)$ & 0.034 \\
\hline
\end{tabular}


Table 1 (continued)

\begin{tabular}{|c|c|c|c|c|}
\hline & Total population $(n=798)$ & Without event $(n=618)$ & With event $(n=180)$ & $P$ value \\
\hline \multicolumn{5}{|l|}{ Post-discharge medication, n (\%) } \\
\hline ACEI & $234(29.3)$ & $177(28.6)$ & $57(31.7)$ & 0.433 \\
\hline ARB & $384(48.1)$ & $294(47.6)$ & $90(50.0)$ & 0.566 \\
\hline DAPT & $796(99.7)$ & $617(99.8)$ & $179(99.4)$ & 0.934 \\
\hline DAPT interruption in 12 months & $12(1.5)$ & $9(1.5)$ & $3(1.7)$ & 0.838 \\
\hline Aspirin & 797 (99.9) & $617(99.8)$ & $180(100.0)$ & 0.589 \\
\hline Clopidogrel & $797(99.9)$ & $618(100.0)$ & $179(99.4)$ & 0.226 \\
\hline$\beta$-blocker & $744(93.2)$ & $579(93.7)$ & $165(91.7)$ & 0.342 \\
\hline Statins & $787(98.6)$ & $611(98.9)$ & $176(97.8)$ & 0.459 \\
\hline Statins interruption in 12 months & $31(3.9)$ & $21(3.4)$ & $10(5.6)$ & 0.187 \\
\hline Proton pump inhibitor & $790(99.0)$ & $613(99.2)$ & $177(98.3)$ & 0.554 \\
\hline Oral hypoglycemic agents & $409(51.3)$ & $321(51.9)$ & $88(48.9)$ & 0.471 \\
\hline Metformin & $167(20.9)$ & $135(21.8)$ & $32(17.8)$ & 0.238 \\
\hline Alpha-glucosidase inhibitor & $181(22.7)$ & $137(22.2)$ & $44(24.4)$ & 0.521 \\
\hline Sulfonylurea & $123(15.4)$ & $99(16.0)$ & $24(13.3)$ & 0.380 \\
\hline Dipeptidyl peptidase 4 inhibitor & $15(1.9)$ & $12(1.9)$ & $3(1.7)$ & 0.811 \\
\hline Insulin & $217(27.2)$ & $156(25.2)$ & $61(33.9)$ & 0.022 \\
\hline \multicolumn{5}{|l|}{ Angiographic data } \\
\hline LM disease, n (\%) & $44(5.5)$ & $22(3.6)$ & $22(12.2)$ & $<0.001$ \\
\hline One-vessel disease, n (\%) & $167(20.9)$ & $146(23.6)$ & $21(11.7)$ & 0.001 \\
\hline Two-vessel disease, n (\%) & $287(36.0)$ & $233(37.7)$ & $54(30.0)$ & 0.058 \\
\hline Three-vessel disease, n (\%) & $344(43.1)$ & $239(38.7)$ & $105(58.3)$ & $<0.001$ \\
\hline Chronic total occlusion, n (\%) & $117(14.7)$ & $67(10.8)$ & $50(27.8)$ & $<0.001$ \\
\hline Diffuse lesion, n (\%) & $237(29.7)$ & $169(27.3)$ & $68(37.8)$ & 0.007 \\
\hline Bifurcation lesion, n (\%) & $186(23.3)$ & $124(20.1)$ & $62(34.4)$ & $<0.001$ \\
\hline In-stent restenosis, n (\%) & $58(7.3)$ & $38(6.1)$ & $20(11.1)$ & 0.024 \\
\hline SYNTAX score & $12.0 \pm 5.5$ & $11.1 \pm 5.1$ & $15.2 \pm 6.0$ & $<0.001$ \\
\hline \multicolumn{5}{|l|}{ Procedural results } \\
\hline \multicolumn{5}{|l|}{ Target vessel territory, n (\%) } \\
\hline LM & $25(3.1)$ & $14(2.3)$ & $11(6.1)$ & 0.009 \\
\hline LAD & $513(64.3)$ & $393(63.6)$ & $120(66.7)$ & 0.449 \\
\hline LCX & $335(42.0)$ & $249(40.3)$ & $86(47.8)$ & 0.073 \\
\hline RCA & $398(49.9)$ & $300(48.5)$ & $98(54.4)$ & 0.164 \\
\hline DES implantation, n (\%) & $785(98.4)$ & $608(98.4)$ & $177(98.3)$ & 0.964 \\
\hline DCB use, n (\%) & $15(1.9)$ & $10(1.6)$ & $5(2.8)$ & 0.313 \\
\hline Complete revascularization, $\mathrm{n}(\%)$ & $414(51.9)$ & $333(53.9)$ & $81(45.0)$ & 0.036 \\
\hline Number of stents & $2.1 \pm 1.3$ & $2.0 \pm 1.2$ & $2.4 \pm 1.5$ & 0.001 \\
\hline
\end{tabular}

Italic values indicate statistically significant associations

$B M I$ body mass index, SBP systolic blood pressure, $D B P$ diastolic blood pressure, $C A D$ coronary artery disease, $M I$ myocardial infarction, $P C I$ percutaneous coronary intervention, $P V D$ peripheral vascular disease, $T G$ s triglycerides, $T C$ total cholesterol, $L D L-C$ low-density lipoprotein cholesterol, $H D L-C$ high-density lipoprotein cholesterol, $h$ s-CRP high-sensitivity C-reactive protein, eGFR estimated glomerular filtration rate, FBG fasting blood glucose, HbA1c glycosylated hemoglobin A1c, TyG triglyceride glucose, LVEF left ventricular ejection fraction, UA unstable angina, NSTEMI non-ST-segment elevation myocardial infarction, ACEI angiotensin converting enzyme inhibitor, ARB angiotensin receptor blocker, DAPT dual antiplatelet therapy, LM left main artery, SYNTAX synergy between PCI with taxus and cardiac surgery, $L A D$ left anterior descending artery, $L C X$ left circumflex artery, $R C A$ right coronary artery, $D E S$ drug-eluting stent, $D C B$ drug-coated balloon

\section{Correlation between the TyG index and cardiovascular risk factors}

The Spearman's rank or Pearson correlation analysis was performed to determine the correlation between the TyG index and traditional or commonly-used risk factors for cardiovascular disease. The TyG index was positively correlated with BMI, FBG, HbA1c, TGs, TC, LDL-C, uric acid, and hs-CRP, while negatively correlated with age and HDL-C (Table 3). 
Table 2 Baseline clinical characteristics of patients stratified by the optimal cutoff point of TyG index

\begin{tabular}{|c|c|c|c|c|}
\hline & Total population $(n=798)$ & $\begin{array}{l}\text { Lower TyG index }(<9.18 \\
\mathrm{n}=429)\end{array}$ & $\begin{array}{l}\text { Higher TyG index ( } \geq 9.18 \\
n=369 \text { ) }\end{array}$ & $P$ value \\
\hline Age, years & $60.9 \pm 8.3$ & $62.1 \pm 7.9$ & $59.5 \pm 8.5$ & $<0.001$ \\
\hline Sex, male, n (\%) & $545(68.3)$ & $303(70.6)$ & $242(65.6)$ & 0.127 \\
\hline $\mathrm{BMI}, \mathrm{kg} / \mathrm{m}^{2}$ & $26.7 \pm 3.2$ & $26.3 \pm 3.2$ & $27.1 \pm 3.2$ & $<0.001$ \\
\hline Heart rate, bpm & $71.7 \pm 10.2$ & $70.9 \pm 9.6$ & $72.5 \pm 10.8$ & 0.028 \\
\hline $\mathrm{SBP}, \mathrm{mmHg}$ & $131.8 \pm 17.1$ & $131.3 \pm 16.8$ & $132.2 \pm 17.5$ & 0.443 \\
\hline $\mathrm{DBP}, \mathrm{mmHg}$ & $76.8 \pm 10.1$ & $76.2 \pm 10.1$ & $77.6 \pm 10.1$ & 0.051 \\
\hline Smoking, n (\%) & $417(52.3)$ & $227(52.9)$ & $190(51.5)$ & 0.688 \\
\hline Drinking, n (\%) & $184(23.1)$ & $106(24.7)$ & $78(21.1)$ & 0.233 \\
\hline Family history of CAD, n (\%) & $93(11.7)$ & $44(10.3)$ & 49 (13.3) & 0.185 \\
\hline Duration of diabetes, years & $8.2 \pm 4.3$ & $8.3 \pm 4.3$ & $8.2 \pm 4.2$ & 0.681 \\
\hline \multicolumn{5}{|l|}{ Medical history, n (\%) } \\
\hline Hypertension & $573(71.8)$ & $303(70.6)$ & $270(73.2)$ & 0.426 \\
\hline Dyslipidemia & $710(89.0)$ & $347(80.9)$ & $363(98.4)$ & $<0.001$ \\
\hline Prior Ml & $175(21.9)$ & $90(21.0)$ & $85(23.0)$ & 0.484 \\
\hline Prior $\mathrm{PCl}$ & $151(18.9)$ & $82(19.1)$ & $69(18.7)$ & 0.881 \\
\hline Prior stroke & $109(13.7)$ & $60(14.0)$ & 49 (13.3) & 0.772 \\
\hline Prior PVD & $125(15.7)$ & $72(16.8)$ & $53(14.4)$ & 0.348 \\
\hline \multicolumn{5}{|l|}{ Laboratory results } \\
\hline $\mathrm{TGs}, \mathrm{mg} / \mathrm{dL}$ & $138.2(97.2,198.5)$ & $99.2(78.4,127.1)$ & $204.7(164.4,276.0)$ & $<0.001$ \\
\hline $\mathrm{TC}, \mathrm{mg} / \mathrm{dL}$ & $157.2 \pm 39.7$ & $145.9 \pm 35.0$ & $170.4 \pm 40.8$ & $<0.001$ \\
\hline $\mathrm{LDL}-\mathrm{C}, \mathrm{mg} / \mathrm{dL}$ & $94.2 \pm 33.1$ & $88.2 \pm 30.5$ & $101.1 \pm 34.6$ & $<0.001$ \\
\hline $\mathrm{HDL}-\mathrm{C}, \mathrm{mg} / \mathrm{dL}$ & $36.9 \pm 8.7$ & $38.7 \pm 9.3$ & $34.8 \pm 7.3$ & $<0.001$ \\
\hline hs-CRP, mg/L & $1.6(0.7,4.1)$ & $1.3(0.6,4.2)$ & $1.9(0.9,3.9)$ & 0.009 \\
\hline Creatinine, mg/dL & $0.8 \pm 0.2$ & $0.8 \pm 0.2$ & $0.8 \pm 0.2$ & 0.655 \\
\hline $\mathrm{eGFR}, \mathrm{mL} /\left(\mathrm{min}^{*} 1.73 \mathrm{~m}^{2}\right)$ & $96.5 \pm 21.6$ & $96.6 \pm 21.3$ & $96.5 \pm 22.0$ & 0.992 \\
\hline Uric acid, $\mu \mathrm{mol} / \mathrm{L}$ & $328.0 \pm 75.6$ & $322.2 \pm 74.9$ & $334.8 \pm 75.9$ & 0.019 \\
\hline $\mathrm{FBG}, \mathrm{mg} / \mathrm{dL}$ & $127.7(109.6,157.0)$ & $115.4(102.3,133.1)$ & $149.9(125.3,177.8)$ & $<0.001$ \\
\hline $\mathrm{HbA1c}, \%$ & $7.5 \pm 1.3$ & $7.1 \pm 1.2$ & $7.8 \pm 1.3$ & $<0.001$ \\
\hline TyG index & $9.1 \pm 0.6$ & $8.6 \pm 0.4$ & $9.7 \pm 0.4$ & $<0.001$ \\
\hline LVEF, \% & $64.0 \pm 6.6$ & $64.0 \pm 6.8$ & $64.0 \pm 6.4$ & 0.986 \\
\hline Initial diagnosis, n (\%) & & & & 0.022 \\
\hline UA & $650(81.5)$ & $362(84.4)$ & $288(78.0)$ & \\
\hline NSTEMI & $148(18.5)$ & $67(15.6)$ & $81(22.0)$ & \\
\hline \multicolumn{5}{|l|}{ Pre-admission medication, n (\%) } \\
\hline ACEI & $79(9.9)$ & $44(10.3)$ & $35(9.5)$ & 0.716 \\
\hline ARB & $128(16.0)$ & $66(15.4)$ & $62(16.8)$ & 0.586 \\
\hline DAPT & $253(31.7)$ & $136(31.7)$ & $117(31.7)$ & 0.999 \\
\hline Aspirin & $427(53.5)$ & $226(52.7)$ & $201(54.5)$ & 0.613 \\
\hline Clopidogrel & $264(33.1)$ & $141(32.9)$ & $123(33.3)$ & 0.889 \\
\hline$\beta$-blocker & $166(20.8)$ & $92(21.4)$ & $74(20.1)$ & 0.629 \\
\hline Statins & $233(29.2)$ & $127(29.6)$ & $106(28.7)$ & 0.786 \\
\hline Proton pump inhibitor & $8(1.0)$ & $4(0.9)$ & $4(1.1)$ & 0.830 \\
\hline Oral hypoglycemic agents & $413(51.8)$ & $220(51.3)$ & $193(52.3)$ & 0.773 \\
\hline Metformin & $170(21.3)$ & $101(23.5)$ & $69(18.7)$ & 0.096 \\
\hline Alpha-glucosidase inhibitor & $185(23.2)$ & $100(23.3)$ & $85(23.0)$ & 0.927 \\
\hline Sulfonylurea & $126(15.8)$ & $67(15.6)$ & $59(16.0)$ & 0.886 \\
\hline Dipeptidyl peptidase 4 inhibitor & $15(1.9)$ & $7(1.6)$ & $8(2.2)$ & 0.578 \\
\hline Insulin & $225(28.2)$ & $109(25.4)$ & $116(31.4)$ & 0.059 \\
\hline
\end{tabular}


Table 2 (continued)

Total population $(n=798)$

Lower TyG index (<9.18; Higher TyG index ( $\geq 9.18 ; \quad P$ value

$\mathrm{n}=429$ ) $\mathrm{n}=369$ )

\begin{tabular}{|c|c|c|c|c|}
\hline \multicolumn{5}{|l|}{ Post-discharge medication, n (\%) } \\
\hline ACEI & $234(29.3)$ & $114(26.6)$ & $120(32.5)$ & 0.066 \\
\hline ARB & $384(48.1)$ & $204(47.6)$ & $180(48.8)$ & 0.729 \\
\hline DAPT & $796(99.7)$ & $429(100.0)$ & $367(99.5)$ & 0.214 \\
\hline DAPT interruption in 12 months & $12(1.5)$ & $7(1.6)$ & $5(1.4)$ & 0.749 \\
\hline Aspirin & 797 (99.9) & $429(100.0)$ & $368(99.7)$ & 0.462 \\
\hline Clopidogrel & $797(99.9)$ & $429(100.0)$ & $368(99.7)$ & 0.462 \\
\hline$\beta$-blocker & $744(93.2)$ & $400(93.2)$ & $344(93.2)$ & 0.993 \\
\hline Statins & $787(98.6)$ & $423(98.6)$ & $364(98.6)$ & 0.958 \\
\hline Statins interruption in 12 months & $31(3.9)$ & $14(3.3)$ & $17(4.6)$ & 0.327 \\
\hline Proton pump inhibitor & $790(99.0)$ & $426(99.3)$ & $364(98.6)$ & 0.568 \\
\hline Oral hypoglycemic agents & $409(51.3)$ & $217(50.6)$ & $192(52.0)$ & 0.683 \\
\hline Metformin & $167(20.9)$ & $98(22.8)$ & $69(18.7)$ & 0.151 \\
\hline Alpha-glucosidase inhibitor & $181(22.7)$ & $97(22.6)$ & $84(22.8)$ & 0.959 \\
\hline Sulfonylurea & $123(15.4)$ & $64(14.9)$ & $59(16.0)$ & 0.676 \\
\hline Dipeptidyl peptidase 4 inhibitor & $15(1.9)$ & $7(1.6)$ & $8(2.2)$ & 0.578 \\
\hline Insulin & $217(27.2)$ & $104(24.2)$ & $113(30.6)$ & 0.043 \\
\hline \multicolumn{5}{|l|}{ Angiographic data } \\
\hline LM disease, $n(\%)$ & $44(5.5)$ & $19(4.4)$ & $25(6.8)$ & 0.148 \\
\hline One-vessel disease, n (\%) & $167(20.9)$ & $92(21.4)$ & $75(20.3)$ & 0.698 \\
\hline Two-vessel disease, n (\%) & $287(36.0)$ & $163(38.0)$ & $124(33.6)$ & 0.197 \\
\hline Three-vessel disease, n (\%) & $344(43.1)$ & $174(40.6)$ & $170(46.1)$ & 0.117 \\
\hline Chronic total occlusion, n (\%) & $117(14.7)$ & $55(12.8)$ & $62(16.8)$ & 0.113 \\
\hline Diffuse lesion, n (\%) & $237(29.7)$ & $123(28.7)$ & $114(30.9)$ & 0.493 \\
\hline Bifurcation lesion, n (\%) & $186(23.3)$ & $93(21.7)$ & $93(25.2)$ & 0.240 \\
\hline In-stent restenosis, n (\%) & $58(7.3)$ & $28(6.5)$ & $30(8.1)$ & 0.384 \\
\hline SYNTAX score & $12.0 \pm 5.5$ & $11.6 \pm 5.5$ & $12.6 \pm 5.6$ & 0.010 \\
\hline \multicolumn{5}{|l|}{ Procedural results } \\
\hline \multicolumn{5}{|l|}{ Target vessel territory, n (\%) } \\
\hline LM & $25(3.1)$ & $14(3.3)$ & $11(3.0)$ & 0.819 \\
\hline LAD & $513(64.3)$ & $274(63.9)$ & $239(64.8)$ & 0.791 \\
\hline LCX & $335(42.0)$ & $185(43.1)$ & $150(40.7)$ & 0.480 \\
\hline RCA & $398(49.9)$ & $211(49.2)$ & $187(50.7)$ & 0.674 \\
\hline DES implantation, n (\%) & $785(98.4)$ & $425(99.1)$ & $360(97.6)$ & 0.163 \\
\hline DCB use, n (\%) & $15(1.9)$ & $5(1.2)$ & $10(2.7)$ & 0.109 \\
\hline Complete revascularization, $\mathrm{n}(\%)$ & $414(51.9)$ & $229(53.4)$ & $185(50.1)$ & 0.360 \\
\hline Number of stents & $2.1 \pm 1.3$ & $2.1 \pm 1.3$ & $2.1 \pm 1.3$ & 0.700 \\
\hline
\end{tabular}

The groups were stratified by the optimal cutoff point of TyG index determined by ROC curve analysis

Italic values indicate statistically significant associations

$B M I$ body mass index, $S B P$ systolic blood pressure, $D B P$ diastolic blood pressure, $C A D$ coronary artery disease, $M I$ myocardial infarction, $P C / p e r c u t a n e o u s$ coronary intervention, PVD peripheral vascular disease, TGs triglycerides, $T C$ total cholesterol, $L D L-C$ low-density lipoprotein cholesterol, $H D L-C$ high-density lipoprotein cholesterol, $h s-C R P$ high-sensitivity C-reactive protein, eGFR estimated glomerular filtration rate, $F B G$ fasting blood glucose, $H b A 1 c$ glycosylated hemoglobin A1c, TyG triglyceride glucose, LVEF left ventricular ejection fraction, UA unstable angina, NSTEMI non-ST-segment elevation myocardial infarction, ACEI angiotensin converting enzyme inhibitor, $A R B$ angiotensin receptor blocker, DAPT dual antiplatelet therapy, LM left main artery, SYNTAX synergy between PCI with taxus and cardiac surgery, $L A D$ left anterior descending artery, $L C X$ left circumflex artery, $R C A$ right coronary artery, $D E S$ drug-eluting stent, $D C B$ drug-coated balloon

\section{Clinical outcomes and Kaplan-Meier analysis}

During the 36-month follow-up period, $180(22.6 \%)$ endpoint events including $14(1.8 \%)$ all-cause death, 37 (4.6\%) non-fatal MI, and 129 (16.2\%) ischemia-driven revascularization were documented to perform the present analyses. The incidence of adverse prognosis was compared between groups stratified by the optimal cutoff point of TyG index determined by ROC curve analysis. 


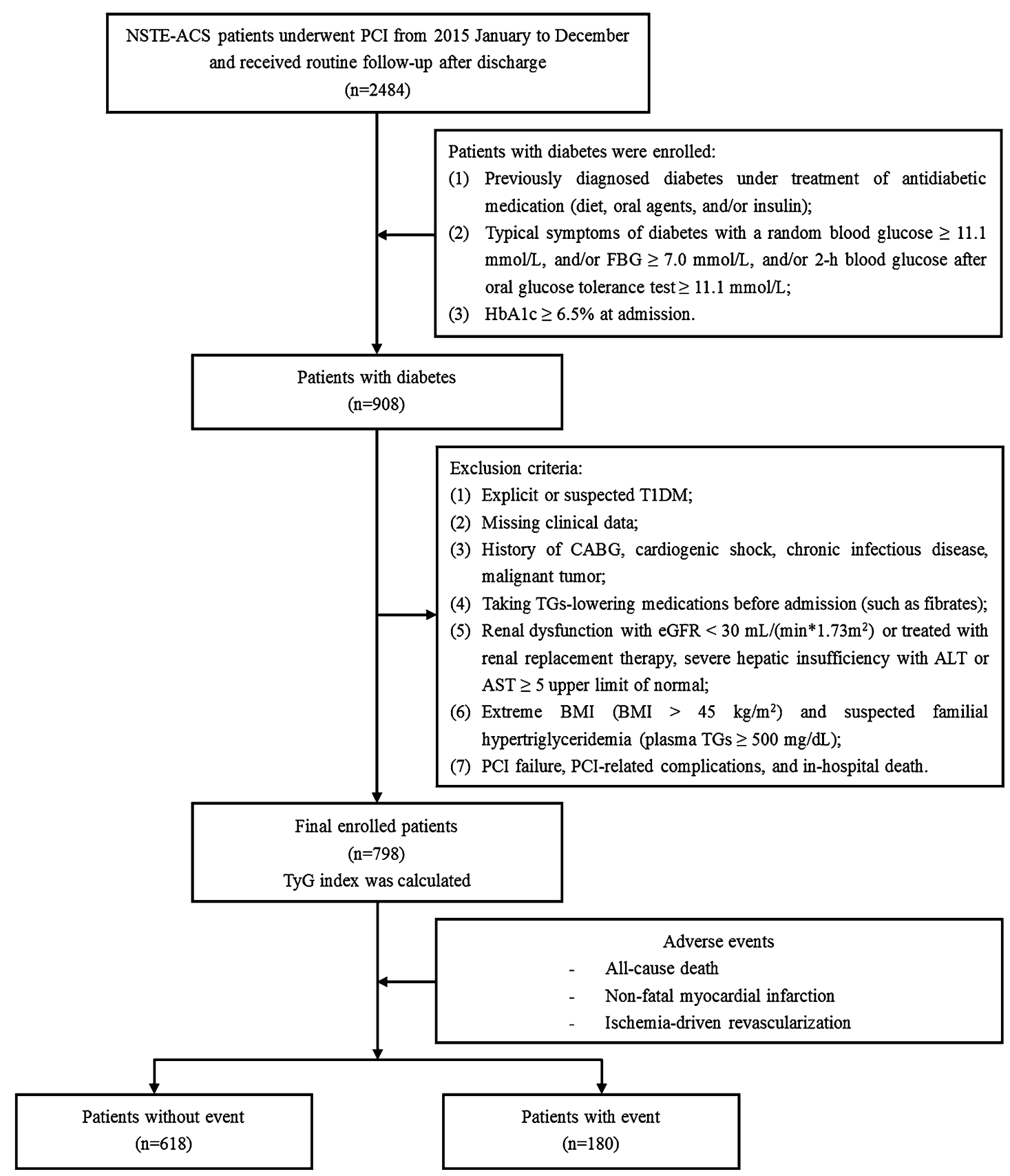

Fig. 1 Flow chart of the study population enrollment. NSTE-ACS non-ST-segment elevation acute coronary syndrome, PCI percutaneous coronary intervention, FBG fasting blood glucose, HbA1c glycosylated hemoglobin A1C, T1DM type 1 diabetes mellitus, CABG coronary artery bypass grafting, TGs triglycerides, eGFR estimated glomerular filtration rate, ALT alanine transaminase, AST aspartate transaminase, BMI body mass index, TyG triglyceride glucose

The incidence of primary endpoint, non-fatal MI and ischemia-driven revascularization increased significantly in patients with higher TyG index compared with those with lower TyG index (all chi-square $\mathrm{P}<0.001$ ). However, the all-cause death rate was similar between the two groups (chi-square $\mathrm{P}=0.172$ ) (Table 4$)$.
Kaplan-Meier curves for incidence of primary endpoint and each component of it according to the optimal cutoff point of TyG index were shown in Fig. 2. KaplanMeier curves for primary endpoint showed a significant difference between the lower and higher TyG index group (Fig. 2a, Log-rank $\mathrm{P}<0.001$ ). The difference was mainly 


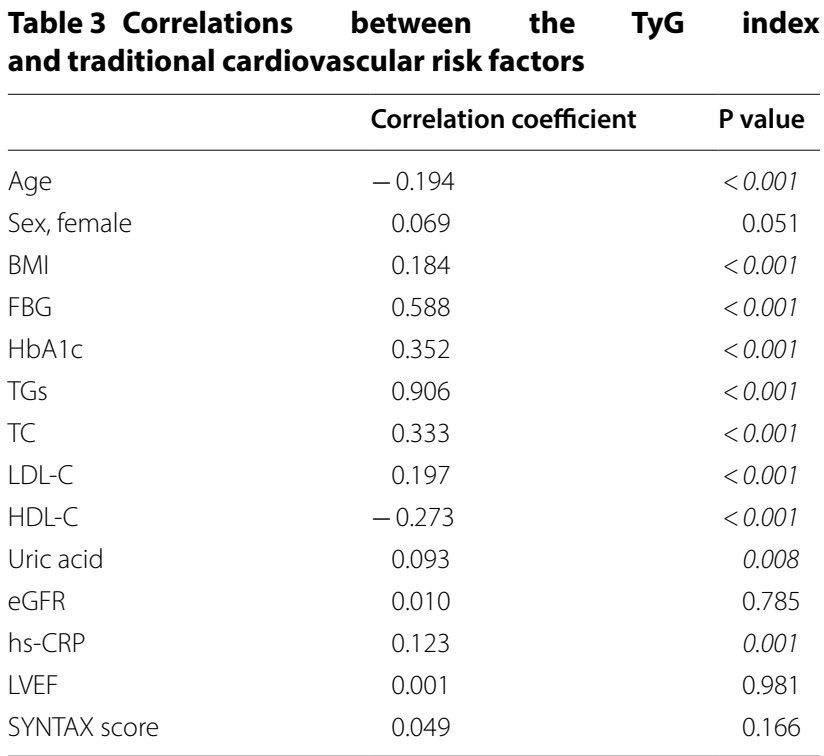

Italic values indicate statistically significant associations

$B M /$ body mass index, FBG fasting blood glucose, HbA1c glycosylated hemoglobin A1C, TGs triglycerides, TC total cholesterol, LDL-C low-density lipoprotein cholesterol, HDL-C high-density lipoprotein cholesterol, eGFR estimated glomerular filtration rate, $h s-C R P$ high-sensitivity C-reactive protein, LVEF left ventricular ejection fraction, SYNTAX synergy between PCI with taxus and cardiac surgery

Table 4 Incidence of endpoint events according to the optimal cutoff point of TyG index

\begin{tabular}{llll}
\hline & $\begin{array}{l}\text { Lower TyG index } \\
(<\mathbf{9 . 1 8 ;} \mathbf{n = 4 2 9 )}\end{array}$ & $\begin{array}{l}\text { Higher TyG index } \\
(\mathbf{\geq 9 . 1 8 ;} \mathbf{n = 3 6 9 )}\end{array}$ & P value \\
\hline $\begin{array}{l}\text { Primary endpoint, } \\
\text { n (\%) }\end{array}$ & $41(9.6)$ & $139(37.7)$ & $<0.001$ \\
$\begin{array}{l}\text { All-cause death, n (\%) } \\
\text { Non-fatal MI, n (\%) }\end{array}$ & $5(1.2)$ & $9(2.4)$ & 0.172 \\
$\begin{array}{l}\text { Ischemia-driven revas- } \\
\text { cularization, n (\%) }\end{array}$ & $27(6.3)$ & $28(7.6)$ & $<0.001$ \\
\hline
\end{tabular}

The groups were stratified by the optimal cutoff point of TyG index determined by ROC curve analysis

Italic values indicate statistically significant associations

Ty $G$ triglyceride glucose, $M I$ myocardial infarction

driven by the increased incidence of non-fatal MI and ischemia-driven revascularization (Fig. 2c, d, both Logrank $\mathrm{P}<0.001)$. Kaplan-Meier curves for all-cause death between the lower and higher TyG index group failed to reach statistical significance (Fig. $2 b$, Log-rank $\mathrm{P}=0.167$ ).

\section{Cox proportional hazard analyses to evaluate the prognostic implication of TyG index}

In multivariate Cox proportional hazard analysis, four models (Model 1-4 as described above) including variables that had statistical significance $(\mathrm{P}<0.2)$ and/or clinical importance were constructed to evaluate the predictive potential of TyG index for primary endpoint. After adjusting for confounding variables, higher TyG index remained to be an independent risk predictor of primary endpoint, despite of regarding TyG index as a nominal or continuous variable (all $\mathrm{P}<0.001$ in Model 1-4) (Table 5). The detailed information of Model 4 was shown in Additional file 1: Table S1.

The predictive value of TyG index for each component of primary endpoint was also evaluated by using model 4. The results showed that a 1-unit increase of TyG index was independently associated with higher risk of nonfatal MI and ischemia-driven revascularization [HR (95\% CI) for non-fatal MI: 3.332 (1.730-6.415), $\mathrm{P}<0.001$; HR (95\% CI) for ischemia-driven revascularization: 3.021 (2.167-4.211), $\mathrm{P}<0.001]$. However, higher TyG index levels failed to be a predictor of all-cause death, which was consistent with the results of Kaplan-Meier curves (Table 6).

Further evaluation of the risk stratification value of TyG index for primary endpoint was performed in various subclasses of the study population. Increased TyG index (per 1-unit) was consistently related to primary endpoint in various subgroups, including age $\leq 65$ or $>65$ years, female or male, BMI $\leq 28$ or $>28 \mathrm{~kg} / \mathrm{m}^{2}$, with or without hypertension, UA or NSTEMI, HbA1c $\leq 7$ or $>7 \%$, $\mathrm{LDL}-\mathrm{C} \leq 70$ or $>70 \mathrm{mg} / \mathrm{dL}$, with or without pre-admission medication including statins, oral hypoglycemic agents and insulin (Fig. 3). Interestingly, the predictive value of TyG index seemed to be more prominent in patients with BMI $>28 \mathrm{~kg} / \mathrm{m}^{2}$ [HR $(95 \% \mathrm{CI}) \mathrm{BMI}>28 \mathrm{~kg} /$ $\mathrm{m}^{2} 5.513(3.631-8.370)$ vs. $\mathrm{BMI} \leq 28 \mathrm{~kg} / \mathrm{m}^{2} 2.178(1.524-$ 3.111 ), $\mathrm{P}$ for interaction $<0.001$ ] and without pre-admission insulin therapy [HR (95\% CI) without insulin 4.011 (2.827-5.691) vs. with insulin 2.255 (1.461-3.479), P for interaction $=0.024]$ (Fig. 3).

\section{Incremental effect of TyG index on predictive value for adverse prognosis}

The addition of TyG index had a significant incremental effect on the AUC obtained from baseline risk model that consisted of risk factors including age, sex (female), smoking, SBP, prior MI, prior PCI, TC, HDLC, eGFR, LVEF, SYNTAX score, LM treatment, complete revascularization, number of stents and statins at discharge (AUC: baseline risk model, 0.800 vs. baseline risk model + TyG index, $0.856, \mathrm{P}$ for comparison $<0.001$ ) (Table 7, Fig. 4d). Moreover, the addition of TyG index significantly improved the reclassification and discrimination ability beyond the baseline risk model with a category-free NRI of 0.346 and an IDI of 0.087 (both $\mathrm{P}<0.001$ ) (Table 8). Adding TGs to the baseline risk model also had a significant incremental effect on prognostic prediction (AUC: baseline risk 


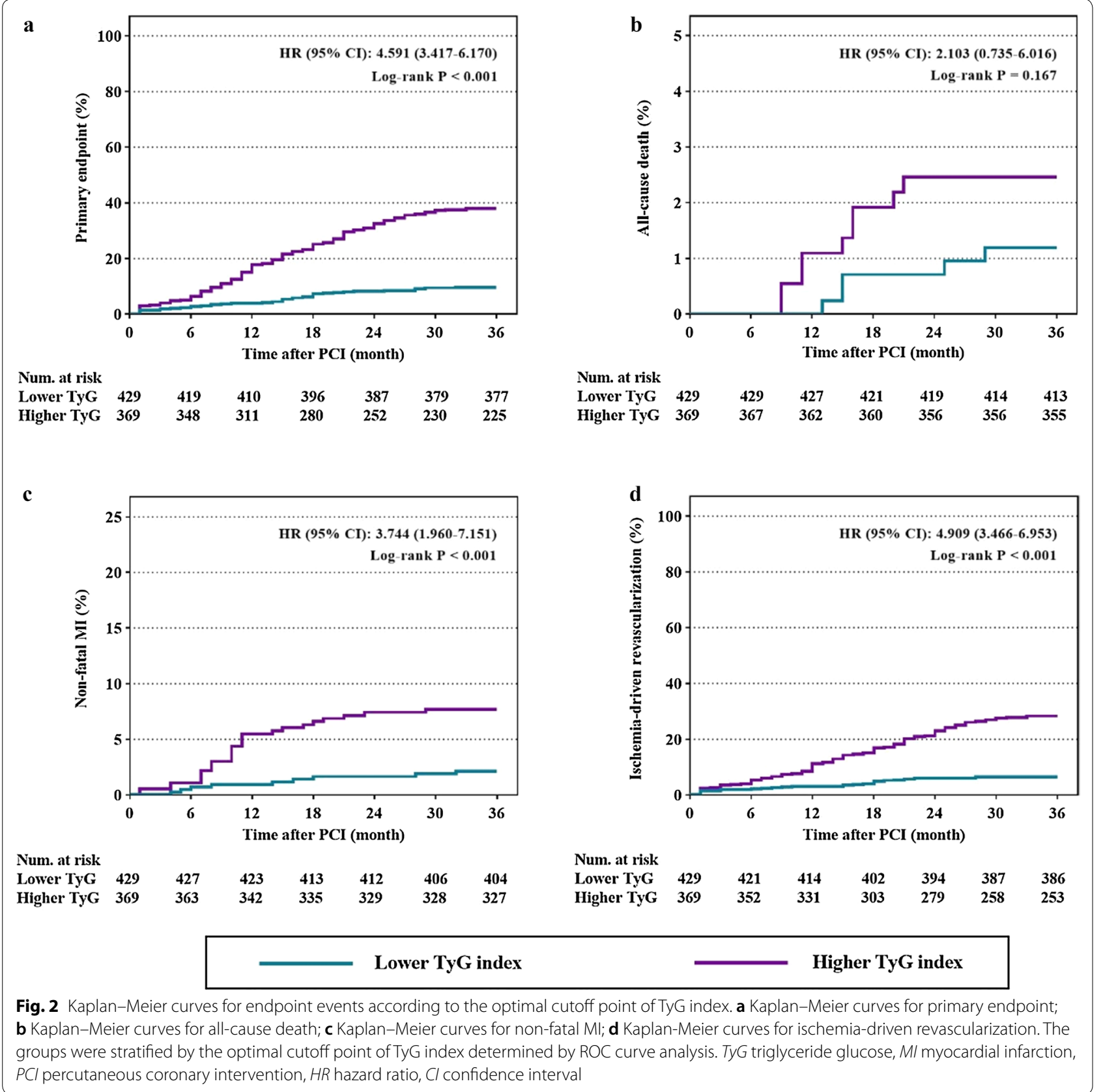

model, 0.800 vs. baseline risk model + TGs, 0.842 , $\mathrm{P}$ for comparison < 0.001; category-free NRI: 0.318, P $<0.001$; IDI: $0.067, \mathrm{P}<0.001$ ) (Tables 7 and 8 , Fig. 4c). However, the addition of glycemic index including FBG or HbA1c did not have a significant incremental effect on the AUC of the baseline risk model (Table 7, Fig. 4a, b). A significant but relatively minor incremental effect on the reclassification and discrimination ability was found after adding $\mathrm{HbA1c}$ to the baseline risk model (Table 8).

\section{Discussion}

In our present study, we retrospectively investigated the predictive significance of IR assessed by TyG index for adverse prognosis in patients with T2DM and NSTEACS who were treated with PCI. The major findings are listed as follows: (1) the TyG index was significantly correlated with variety of risk factors for cardiovascular disease; (2) compared to participants with lower TyG index, those with higher TyG index had an apparently higher 
Table 5 Predictive value of TyG index for primary endpoint in different Cox proportional hazards models

\begin{tabular}{|c|c|c|c|c|c|c|}
\hline & \multicolumn{3}{|c|}{ TyG index as a nominal variable ${ }^{a}$} & \multicolumn{3}{|c|}{ TyG index as a continuous variable ${ }^{\mathbf{b}}$} \\
\hline & $\mathrm{HR}$ & $95 \% \mathrm{Cl}$ & P value & HR & $95 \% \mathrm{Cl}$ & P value \\
\hline Crude model & 4.610 & $3.253-6.533$ & $<0.001$ & 3.367 & $2.677-4.235$ & $<0.001$ \\
\hline Model 1 & 4.858 & $3.367-7.011$ & $<0.001$ & 3.459 & $2.731-4.381$ & $<0.001$ \\
\hline Model 2 & 3.774 & $2.553-5.580$ & $<0.001$ & 2.900 & $2.194-3.832$ & $<0.001$ \\
\hline Model 3 & 3.994 & $2.699-5.991$ & $<0.001$ & 3.031 & $2.294-4.005$ & $<0.001$ \\
\hline Model 4 & 4.062 & $2.732-6.040$ & $<0.001$ & 3.208 & $2.400-4.289$ & $<0.001$ \\
\hline
\end{tabular}

Model 1: adjusted for age, sex (female), BMI, SBP, DBP, smoking, drinking, duration of diabetes, dyslipidemia, prior MI, PCI, stroke and PVD

Model 2: adjusted for variables included in Model 1 and diagnosis (NSTEMI), TC, HDL-C, eGFR, HbA1c, LVEF

Model 3: adjusted for variables included in Model 2 and SYNTAX score, LM treatment, DCB use, complete revascularization and number of stents

Model 4: adjusted for variables included in Model 3 and DAPT at discharge, DAPT interruption in 12 months, statins at discharge, statins interruption in 12 months, oral hypoglycemic agents (metformin, alpha-glucosidase inhibitor, sulfonylurea, dipeptidyl peptidase 4 inhibitor) at discharge and insulin at discharge

Italic values indicate statistically significant associations

TyG triglyceride glucose, $H R$ hazard ratio, $\mathrm{Cl}$ confidence interval

a The HR was examined regarding lower TyG index as reference (stratified by the optimal cutoff point of TyG index determined by ROC curve analysis)

b The HR was examined by per 1-unit increase of TyG index

Table 6 Predictive value of TyG index for primary endpoint and each component in univariate and multivariate analysis

\begin{tabular}{|c|c|c|c|c|c|c|}
\hline & \multicolumn{3}{|c|}{ Univariate analysis } & \multicolumn{3}{|c|}{ Multivariate analysis ${ }^{c}$} \\
\hline & HR & $95 \% \mathrm{Cl}$ & $P$ value & HR & $95 \% \mathrm{Cl}$ & $P$ value \\
\hline \multicolumn{7}{|l|}{ TyG index as a nominal variable ${ }^{a}$} \\
\hline Primary endpoint & 4.610 & $3.253-6.533$ & $<0.001$ & 4.062 & $2.732-6.040$ & $<0.001$ \\
\hline All-cause death & 2.103 & $0.705-6.276$ & 0.183 & 0.872 & $0.179-4.258$ & 0.866 \\
\hline Non-fatal Ml & 3.744 & $1.767-7.935$ & 0.001 & 2.260 & $0.894-5.715$ & 0.085 \\
\hline Ischemia-driven revascularization & 4.920 & $3.218-7.521$ & $<0.001$ & 4.980 & $3.075-8.067$ & $<0.001$ \\
\hline \multicolumn{7}{|l|}{ TyG index as a continuous variable ${ }^{b}$} \\
\hline Primary endpoint & 3.367 & $2.677-4.235$ & $<0.001$ & 3.208 & $2.400-4.289$ & $<0.001$ \\
\hline All-cause death & 1.358 & $0.610-3.024$ & 0.454 & 0.429 & $0.111-1.659$ & 0.220 \\
\hline Non-fatal Ml & 4.449 & $2.684-7.373$ & $<0.001$ & 3.332 & $1.730-6.415$ & $<0.001$ \\
\hline Ischemia-driven revascularization & 2.874 & $2.216-3.727$ & $<0.001$ & 3.021 & $2.167-4.211$ & $<0.001$ \\
\hline
\end{tabular}

Italic values indicate statistically significant associations

Ty $G$ triglyceride glucose, $M I$ myocardial infarction, $H R$ hazard ratio, $C l$ confidence interval

a The HR was examined regarding lower TyG index as reference (stratified by the optimal cutoff point of TyG index determined by ROC curve analysis)

b The HR was examined by per 1-unit increase of TyG index

c The multivariate analysis was performed by using Model 4 [adjusted for age, sex (female), BMI, SBP, DBP, smoking, drinking, duration of diabetes, dyslipidemia, prior MI, PCl, stroke, PVD, diagnosis (NSTEMI), TC, HDL-C, eGFR, HbA1c, LVEF, SYNTAX score, LM treatment, DCB use, complete revascularization, number of stents, DAPT at discharge, DAPT interruption in 12 months, statins at discharge, statins interruption in 12 months, oral hypoglycemic agents (metformin, alpha-glucosidase inhibitor, sulfonylurea, dipeptidyl peptidase 4 inhibitor) at discharge and insulin at discharge]

incidence of primary endpoint; (3) the increased level of TyG index was a strong indicator of worse prognosis in the study population, even after adjustment of confounding risk factors; (4) the addition of TyG index to the baseline risk model including traditional risk factors significantly promoted the ability of risk stratification.

T2DM has been widely recognized as the most significant risk factors for cardiovascular disease and it is very common for patients with ACS combined with T2DM.
Certain studies have demonstrated that T2DM is significantly associated with preclinical cardiovascular organ damage, development of CAD, more complex coronary lesions and adverse prognosis [4-6, 29] and the association has been shown to be mediated primarily by IR [30]. It has been proved that IR is significantly related to the development and progression of coronary atherosclerosis $[7,8]$. Therefore, for patients with or at high risk of CAD, quantitative assessment of the extent of IR is of great 


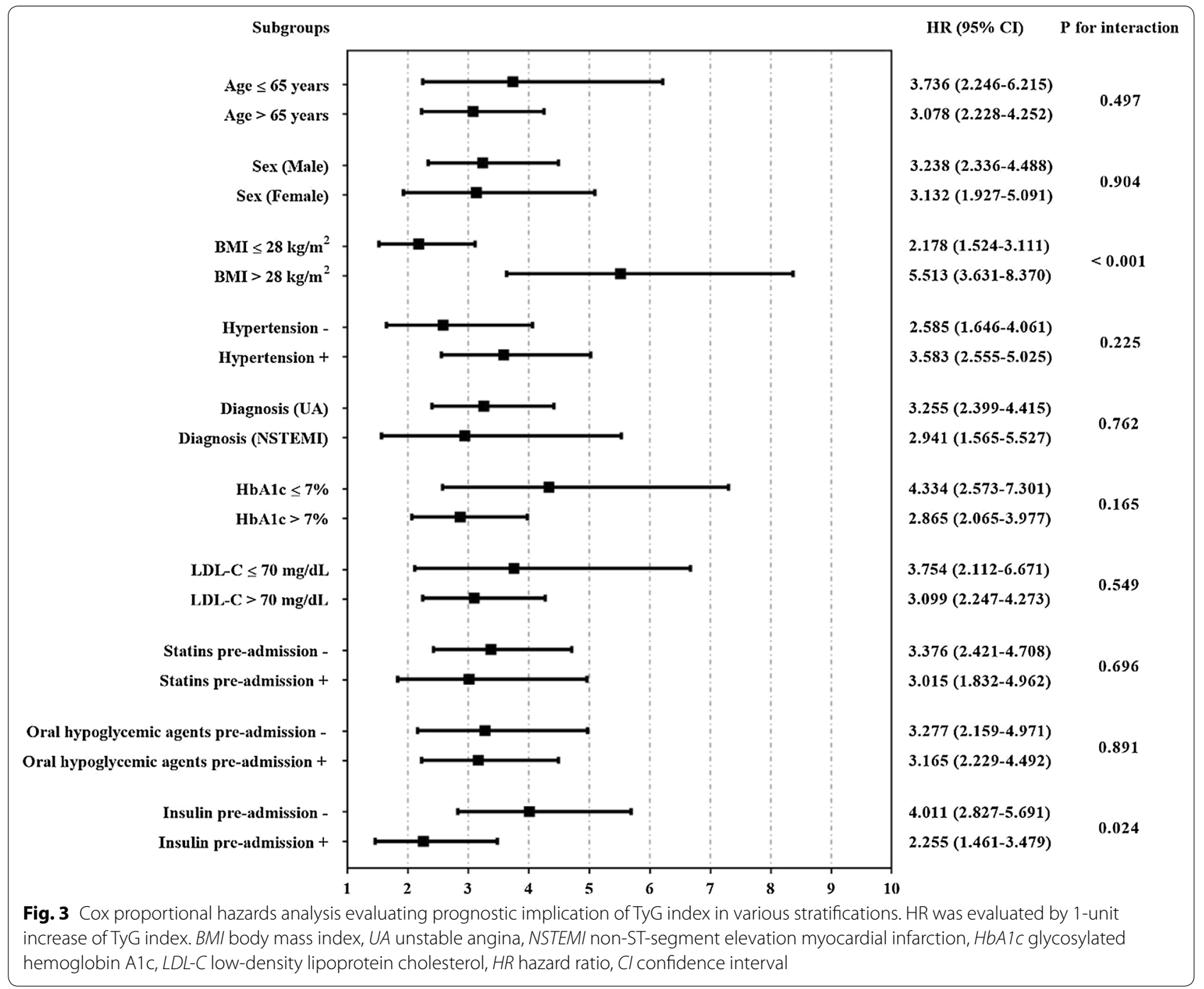

Table 7 C-statistics for discrimination ability of various models

\begin{tabular}{llllll}
\hline & AUC & $\mathbf{9 5 \%} \mathbf{C l}$ & P value & Z value & P for comparison \\
\hline Baseline risk model $^{\mathrm{a}}$ & 0.800 & $0.771-0.827$ & $<0.001$ & Reference & Reference \\
+ FBG & 0.807 & $0.778-0.834$ & $<0.001$ & 1.860 & 0.063 \\
+ HbA1C & 0.811 & $0.782-0.838$ & $<0.001$ & 1.653 & 0.098 \\
+ TGs & 0.842 & $0.815-0.867$ & $<0.001$ & 3.757 & $<0.001$ \\
+ TyG index & 0.856 & $0.829-0.879$ & $<0.001$ & 4.046 & $<0.001$ \\
\hline
\end{tabular}

Italic values indicate statistically significant associations

FBG fasting blood glucose, $\mathrm{HbA} 1 \mathrm{c}$ glycosylated hemoglobin A1c, TGs triglycerides, TyG triglyceride glucose, $A U C$ area under the curve, $\mathrm{Cl}$ confidence interval

a The baseline risk model includes age, sex (female), smoking, SBP, prior MI, prior PCI, TC, HDL-C, eGFR, LVEF, SYNTAX score, LM treatment, complete revascularization, number of stents and statins at discharge

clinical importance for risk stratification and prognosis prediction. The euglycemic-hyperinsulinemic clamp has been acknowledged as the gold standard method for the diagnosis of IR by previous studies [31]. However, this method is relatively time-consuming, expensive and complicated to operate, which makes it comparatively difficult to be applied in real-world clinical practice. Homeostasis model assessment of IR (HOMA-IR), which 

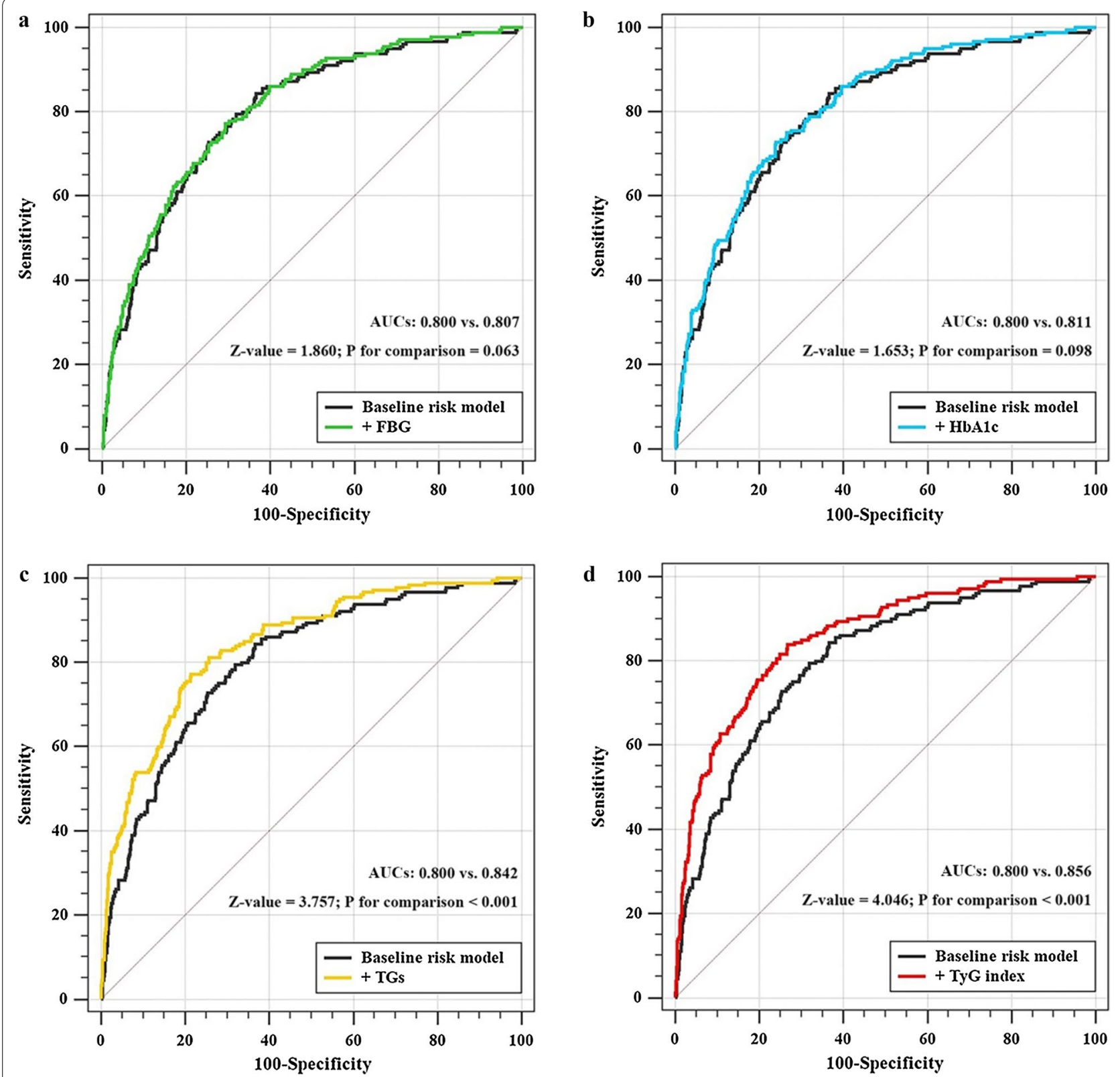

Fig. 4 C-statistics evaluating incremental effect of FBG, HbA1c, TGs or TyG index beyond baseline risk model. a Baseline risk model vs. +FBG; $\mathbf{b}$ baseline risk model vs. +HbA1c; c baseline risk model vs. $+\mathrm{TGs}$; $\mathbf{d}$ baseline risk model vs. + TyG index. The baseline risk model includes age, sex (female), smoking, SBP, prior MI, prior PCI, TC, HDL-C, eGFR, LVEF, SYNTAX score, LM treatment, complete revascularization, number of stents and statins at discharge. FBG fasting blood glucose, HbA1c glycosylated hemoglobin A1C, TGs triglycerides, TyG triglyceride glucose, AUC area under the curve

is calculated by fasting insulin and glucose, has been one of the commonly used method for the assessment of IR in current clinical applications [31]. However, the insulin concentration is not routinely measured in clinical practice, which makes HOMA-IR inappropriate for extensive clinical application. Based on these, a surrogate marker of IR named TyG index derived from commonly used clinical indicators (fasting TGs and glucose) has been proposed and showed to be well related to the euglycemic-hyperinsulinemic clamp and HOMA-IR [11, 32-34]. And studies even showed that the TyG index may have a better performance on the prediction of IR and atherosclerosis compared with HOMA-IR [35, 36]. 
Table 8 Category-free NRI and IDI for the incremental predictive values of various models

\begin{tabular}{|c|c|c|c|c|c|c|}
\hline & \multicolumn{3}{|c|}{ Category-free NRI } & \multicolumn{3}{|l|}{ IDI } \\
\hline & Index & $95 \% \mathrm{Cl}$ & P value & Index & $95 \% \mathrm{Cl}$ & $P$ value \\
\hline Baseline risk model ${ }^{a}$ & - & - & Reference & - & - & Reference \\
\hline$+F B G$ & 0.076 & -0.146 to 0.190 & 0.358 & 0.005 & -0.005 to 0.019 & 0.408 \\
\hline$+\mathrm{HbA} 1 \mathrm{c}$ & 0.145 & $0.050-0.234$ & 0.020 & 0.014 & $0.001-0.038$ & 0.020 \\
\hline$+\mathrm{TGs}$ & 0.318 & $0.143-0.399$ & $<0.001$ & 0.067 & $0.028-0.108$ & $<0.001$ \\
\hline+ TyG index & 0.346 & $0.230-0.430$ & $<0.001$ & 0.087 & $0.039-0.128$ & $<0.001$ \\
\hline
\end{tabular}

FBG fasting blood glucose, $H b A 1 c$ glycosylated hemoglobin A1c, TGs triglycerides, TyG triglyceride glucose, NRI net reclassification improvement, IDI integrated discrimination improvement, $\mathrm{Cl}$ confidence interval

a The baseline risk model includes age, sex (female), smoking, SBP, prior MI, prior PCl, TC, HDL-C, eGFR, LVEF, SYNTAX score, LM treatment, complete revascularization, number of stents and statins at discharge

Previous studies have demonstrated that IR evaluated by TyG index is strongly related to the incidence of diabetes and prediabetic status, suggesting that TyG index may be a considerable predictor for early identifying individuals at high risk of developing diabetes and prediabetes, even performs better than other risk factors such as FBG and weight gain [14-16, 37, 38]. Studies also showed that elevated level of TyG index is prominently associated with an increased risk of developing cardiovascular disease including CAD and ischemic stroke, which suggests evaluation of TyG index might be helpful for identifying people who is susceptible to cardiovascular disease, despite existence of traditional cardiovascular risk factors or not $[17,18,39-41]$. And for patients with stable CAD, TyG index has been demonstrated to be positively related to future adverse clinical outcomes, indicating that TyG index may play an important role in the prediction of clinical prognosis in patients with stable CAD [42, 43]. The clinical significance of TyG index has been increasing as the adverse effects of it on individuals with or at high risk of cardiovascular disease have been elucidated. Evaluation of TyG index may have great clinical importance on risk stratification and therapeutic individuation for these patients.

Several studies have shown that there is an important correlation between TyG index and clinical prognosis in patients with ACS. Study from Mao et al. [44] revealed that the level of TyG index is strongly associated with the complexity of coronary lesions and the incidence of future adverse cardiovascular event during a 12-month of follow-up in patients diagnosed with NSTE-ACS. Another observational study from Luo et al. [45] assessing the predictive potential of TyG index for 1-year prognosis suggested that the increased TyG index might be an effective indicator of worse prognosis in patients with STsegment elevation myocardial infarction (STEMI) who were treated with PCI. However, whether the predictive value of TyG index for poor prognosis was consistent in patients with or without diabetes was not investigated in former studies. Ma et al. [46] evaluated the predictive significance of TyG index in participants with T2DM and ACS undergoing PCI and showed that the TyG index was the independent predictor of adverse clinical outcomes. However, whether the addition of TyG index has an incremental effect on predicting adverse cardiovascular prognosis at the basis of traditional risk factors is not confirmed. The present study, which has a relatively longer follow-up period, revealed the significant prognostic impact of TyG index and its incremental effect on risk stratification at the basis of traditional risk factors in a specific cohort of patients with T2DM and NSTE-ACS undergoing $\mathrm{PCI}$, which makes the study be great agreement and complement to previous literatures.

Based on the formula used for calculating TyG index, it's easy to conclude that the value of TyG index is determined by the levels of fasting TGs and FPG. Therefore, factors manipulating these two indicators such as statins, TGs-lowering therapies and antidiabetic medications all have certain impacts on the evaluation of TyG index [47, 48]. The results of current study showed that the addition of TyG index or fasting TGs, but not FBG, had a significant incremental effect on predictive performance at the basis of a baseline risk model. This may be mainly attributed to the large proportion of participants receiving antidiabetic medications before admission, which can influence the assessment of the true level of FBG, thus further affecting its predictive value for adverse prognosis. The exclusion of patients receiving TGs-lowering therapies mitigated the influence of TGs-lowering medications on fasting TGs levels to great extent, so the fasting TGs levels manifested a significant discriminative performance of predicting adverse prognosis beyond a baseline risk model including traditional risk factors. The stratification analysis according to receiving statins, oral hypoglycemic agents and insulin or not showed that the predictive value of TyG index was more significant in 
patients without insulin treatment before admission [HR (95\% CI) without insulin $4.011(2.827-5.691)$ vs. with insulin 2.255 (1.461-3.479), P for interaction $=0.024$ ], which indicates that the antidiabetic therapies, especially insulin, do have an important effect on predictive performance of TyG index for adverse prognosis.

The potential mechanism inducing the association of IR presented by TyG index with development and progression of cardiovascular disease remains uncertain, several speculations summarize as follows. (1) It has been demonstrated that TyG index is closely related to traditional risk factors for cardiovascular disease such as hypertension [49] and renal insufficiency [50]. In the present study, participants with higher TyG index exactly tended to combine with more severe and complex clinical conditions in terms of BMI, blood pressure, lipid profiles and coronary lesions, and correlation analysis also showed that TyG index is positively related to multiple risk factors for cardiovascular disease. (2) Study have shown that FBG mainly reflects IR from liver, whereas fasting TGs mainly reflects IR from adipose cell [51]. Therefore, it can be concluded that TyG index may reflect IR from two aspects and thus be closely related to IR, which has been widely demonstrated to have significant relationship with endothelial dysfunction, oxidative stress, cardiovascular remodeling, coagulation imbalance and inflammation response [52-54]. Indeed, a positive association between TyG index and hs-CRP levels was confirmed in the present study. (3) Certain studies have also identified an important correlation between TyG index and coronary artery calcification [55], which may be another potential mechanism. (4) The TyG index has been also demonstrated to be related to arterial stiffness evaluated by pulse pressure, brachial-ankle pulse wave velocity and carotid-femoral pulse wave velocity, which has been recognized as cardiovascular risk predictor [10, 56-58].

Since adverse prognostic impacts of IR on individuals with CAD have been elucidated by previous studies, taking assessment and intervention of IR into long-term management strategies may be beneficial for patients with CAD. However, the relative lack of research about intervention on IR in patients with CAD makes it uncertain whether intervention of IR is necessary for the management of such patients. Former studies have shown that whole-grain consumption plays a significant protective role on IR and inflammatory markers $[59,60]$. However, a recent systematic review of 9 RCTs indicated that there is insufficient evidence on the effect of whole-grain diets on cardiovascular outcomes or major cardiovascular disease risk factors [61]. This may be partly attributed to the fact that the association between whole-grain consumption and IR is partially mediated by adiposity [60]. Our present study also revealed that the predictive value of IR presented by TyG index seemed to be more prominent in patients $\mathrm{BMI}>28 \mathrm{~kg} / \mathrm{m}^{2}$ [HR $(95 \% \mathrm{CI})$ $\mathrm{BMI}>28 \mathrm{~kg} / \mathrm{m}^{2} 4.625(2.863-7.471)$ vs. $\mathrm{BMI} \leq 28 \mathrm{~kg} /$ $\mathrm{m}^{2} 2.355$ (1.749-3.170), P for interaction $\left.=0.044\right]$. Further specific-designed studies are required to determine whether interventions of IR assessed by TyG index have a positive impact on improving clinical prognosis in this population.

This study confirmed the predictive value of IR presented by TyG index for adverse prognosis in a cohort including patients with T2DM and NSTE-ACS who were treated with PCI, which indicates that TyG index can be an available predictor in clinical practice and has a positive effect on more comprehensive risk evaluation and stratification on the basis of traditional risk factors in this selected population. Meanwhile, some limitations of the study should be recognized. (1) This study is a single-center, retrospective, observational study in a highly selected cohort with strict exclusion criteria, and the sample size is relatively small, which may weaken the power of the results. Further prospective, multi-center study in a more extensive population with larger sample size are needed to further verify the present findings. (2) The TyG index was assessed only once at admission. The changes of TyG index during the follow-up period, which may have better prediction value for adverse prognosis, were not assessed in our study. (3) Certain proportion of participants received statins therapy and antidiabetic treatment at admission, which may have potential impact on the TyG index and the study results. (4) Non-fatal stroke and cardiovascular death, the commonly-used endpoint events, were not specified in current study since the information about them was relatively inadequate. (5) Nearly all of the study population is Chinese patients. The results should be cautiously interpreted and expanded to Western population as differences in metabolic levels exist among different races. (6) It is hard to rule out that some patients may be complicated with undiagnosed systemic diseases, such as occult malignancies, which may have impact on the assessment of prognosis. (7) The HOMA-IR was not calculated in the present study, so the comparison between TyG index and HOMA-IR is lacking.

\section{Conclusions}

Increased IR extent presented by TyG index is a prominent risk predictor of adverse prognosis in patients with T2DM and NSTE-ACS who were treated with PCI. The addition of the TyG index to a baseline risk model has a strong incremental effect on the predictive potential for adverse prognosis. Further prospective, randomized studies need to be performed to determine whether 
interventions for IR have a positive impact on improving clinical prognosis.

\section{Supplementary information}

Supplementary information accompanies this paper at https://doi. org/10.1186/s12933-020-01086-5.

Additional file 1: Table S1. Univariate and multivariate Cox proportional hazard analysis (model 4) for primary endpoint.

\section{Abbreviations}

CAD: Coronary artery disease; ACS: Acute coronary syndrome; T2DM: Type 2 diabetes mellitus; IR: Insulin resistance; TyG index: Triglyceride-glucose index; NSTE-ACS: Non-ST-segment elevation acute coronary syndrome; PCl: Percutaneous coronary intervention; TGs: Triglycerides; BMl: Body mass index; FBG: Fasting blood glucose; NSTEMI: Non-ST-segment elevation myocardial infarction; UA: Unstable angina; SBP: Systolic blood pressure; DBP: Diastolic blood pressure; HbA1c: Glycosylated hemoglobin A1c; TC: Total cholesterol; LDL-C: Low-density lipoprotein cholesterol; HDL-C: High-density lipoprotein cholesterol; eGFR: Estimated glomerular filtration rate; SYNTAX: The synergy between PCI with taxus and cardiac surgery; MI: Myocardial infarction; PVD: Peripheral vascular disease; LVEF: Left ventricular ejection fraction; DAPT: Dual antiplatelet therapy; ROC: Receiver operating characteristics; hs-CRP: Highsensitivity C-reactive protein; LM: Left main artery; AUC: Area under the curve; HR: Hazard ratio; Cl: Confidence interval; NRI: Net reclassification improvement; IDI: Integrated discrimination improvement; HOMA-IR: Homeostasis model assessment of insulin resistance.
\end{abstract}

\section{Acknowledgements}

Not applicable.

\section{Authors' contributions}

QZ and TYZ made substantial contributions to study design, data collection, data analysis and manuscript writing. YJZ made substantial contributions to study design and intellectual direction. YJC, YM, YKX, JQY made contributions to data collection and analysis. All authors read and approved the final manuscript.

\section{Funding}

This work was supported by the grant from National Key Research and Development Program of China (2017YFC0908800); Beijing Municipal Administration of Hospitals "Ascent Plan" (DFL20150601) and "Mission plan" (SML20180601); Beijing Municipal Health Commission "Project of Science and Technology Innovation Center" (PXM2019_026272_000006) (PXM2019_026272_000005).

\section{Availability of data and materials}

The datasets used and/or analyzed during the current study are available from the corresponding author on reasonable request.

\section{Ethics approval and consent to participate}

Written or oral informed consent was obtained from each participant, and the study protocol was approved by the Clinical Research Ethics Committee of Beijing Anzhen Hospital, Capital Medical University.

\section{Consent for publication}

Not applicable.

\section{Competing interests}

The authors declare that they have no competing interests.

\section{Author details}

${ }^{1}$ Department of Cardiology, Beijing Anzhen Hospital, Capital Medical University, Beijing Institute of Heart Lung and Blood Vessel Disease, Beijing Key Laboratory of Precision Medicine of Coronary Atherosclerotic Disease, Clinical Center for Coronary Heart Disease, Capital Medical University, Beijing 100029, China. ${ }^{2}$ Research Center for Coronary Heart Disease, Fuwai Hospital, National
Center for Cardiovascular Diseases, Chinese Academy of Medical Sciences and Peking Union Medical College, Beijing 100037, China.

Received: 12 May 2020 Accepted: 2 July 2020

Published online: 08 July 2020

\section{References}

1. Jernberg T, Hasvold P, Henriksson M, Hjelm H, Thuresson M, Janzon M. Cardiovascular risk in post-myocardial infarction patients: nationwide real world data demonstrate the importance of a long-term perspective. Eur Heart J. 2015;36(19):1163-70.

2. Fox KAA, Carruthers KF, Dunbar DR, Graham C, Manning JR, De Raedt $\mathrm{H}$, et al. Underestimated and under-recognized: the late consequences of acute coronary syndrome (GRACE UK-Belgian Study). Eur Heart J. 2010;31(22):2755-64.

3. Schwartz GG, Abt M, Bao W, DeMicco D, Kallend D, Miller M, et al. Fasting triglycerides predict recurrent ischemic events in patients with acute coronary syndrome treated with statins. J Am Coll Cardiol. 2015;65(21):2267-75

4. Ray KK, Colhoun HM, Szarek M, Baccara-Dinet M, Bhatt DL, Bittner VA, et al. Effects of alirocumab on cardiovascular and metabolic outcomes after acute coronary syndrome in patients with or without diabetes: a prespecified analysis of the ODYSSEY OUTCOMES randomised controlled trial. Lancet Diabetes Endocrinol. 2019;7(8):618-28.

5. Lenzen M, Ryden L, Ohrvik J, Bartnik M, Malmberg K, Scholte OP, Reimer W, et al. Diabetes known or newly detected, but not impaired glucose regulation, has a negative influence on 1-year outcome in patients with coronary artery disease: a report from the Euro Heart Survey on diabetes and the heart. Eur Heart J. 2006;27(24):2969-74.

6. Maron DJ, Boden WE, Spertus JA, Hartigan PM, Mancini GB, Sedlis SP, et al. Impact of metabolic syndrome and diabetes on prognosis and outcomes with early percutaneous coronary intervention in the COURAGE (Clinical Outcomes Utilizing Revascularization and Aggressive Drug Evaluation) trial. J Am Coll Cardiol. 2011;58(2):131-7.

7. Wu S, Liu W, Ma Q, Yu W, Guo Y, Zhao Y, et al. Association between insulin resistance and coronary plaque vulnerability in patients with acute coronary syndromes: insights from optical coherence tomography. Angiology 2019;70(6):539-46.

8. Iguchi T, Hasegawa T, Otsuka K, Matsumoto K, Yamazaki T, Nishimura S, et al. Insulin resistance is associated with coronary plaque vulnerability: insight from optical coherence tomography analysis. Eur Heart J Cardiovasc Imaging. 2014;15(3):284-91.

9. Uetani T, Amano T, Harada K, Kitagawa K, Kunimura A, Shimbo Y, et al. Impact of insulin resistance on post-procedural myocardial injury and clinical outcomes in patients who underwent elective coronary interventions with drug-eluting stents. JACC Cardiovasc Interv. 2012;5(11):1159-67.

10. Fiorentino TV, Marini MA, Succurro E, Andreozzi F, Sesti G. Relationships of surrogate indexes of insulin resistance with insulin sensitivity assessed by euglycemic hyperinsulinemic clamp and subclinical vascular damage. BMJ Open Diabetes Res Care. 2019;7(1):e911.

11. Guerrero-Romero F, Simental-Mendia LE, Gonzalez-Ortiz M, MartinezAbundis E, Ramos-Zavala MG, Hernandez-Gonzalez SO, et al. The product of triglycerides and glucose, a simple measure of insulin sensitivity. Comparison with the euglycemic-hyperinsulinemic clamp. J Clin Endocrinol Metab. 2010;95(7):3347-51.

12. Mazidi M, Kengne AP, Katsiki N, Mikhailidis DP, Banach M. Lipid accumulation product and triglycerides/glucose index are useful predictors of insulin resistance. J Diabetes Complicat. 2018;32(3):266-70.

13. Du T, Yuan G, Zhang M, Zhou X, Sun X, Yu X. Clinical usefulness of lipid ratios, visceral adiposity indicators, and the triglycerides and glucose index as risk markers of insulin resistance. Cardiovasc Diabetol. 2014;13:146.

14. Brahimaj A, Rivadeneira F, Muka T, Sijbrands EJG, Franco OH, Dehghan A, et al. Novel metabolic indices and incident type 2 diabetes among women and men: the Rotterdam Study. Diabetologia. 2019;62(9):1581-90.

15. Zhang M, Wang B, Liu Y, Sun X, Luo X, Wang C, et al. Cumulative increased risk of incident type 2 diabetes mellitus with increasing triglyceride 
glucose index in normal-weight people: the Rural Chinese Cohort Study. Cardiovasc Diabetol. 2017;16(1):30.

16. Ramírez-Vélez R, Pérez-Sousa MÁ, González-Ruíz K, Cano-Gutierrez CA, Schmidt-RioValle J, Correa-Rodríguez M, et al. Obesity- and lipid-related parameters in the identification of older adults with a high risk of prediabetes according to the American Diabetes Association: an analysis of the 2015 Health, Well-Being, and Aging Study. Nutrients. 2019;11(11):2654

17. Da Silva A, Caldas APS, Hermsdorff HHM, Bersch-Ferreira ÂC, Torreglosa $C R$, Weber $B$, et al. Triglyceride-glucose index is associated with symptomatic coronary artery disease in patients in secondary care. Cardiovasc Diabetol. 2019;18(1):89.

18. Li S, Guo B, Chen H, Shi Z, Li Y, Tian Q, et al. The role of the triglyceride (triacylglycerol) glucose index in the development of cardiovascular events: a retrospective cohort analysis. Sci Rep-Uk. 2019;9(1):7320.

19. Sánchez-Iñigo L, Navarro-González D, Fernández-Montero A, PastranaDelgado J, Martínez JA. The TyG index may predict the development of cardiovascular events. Eur J Clin Invest. 2016;46(2):189-97.

20. Sánchez-Î́igo L, Navarro-González D, Pastrana-Delgado J, FernándezMontero A, Martínez JA. Association of triglycerides and new lipid markers with the incidence of hypertension in a Spanish cohort. J Hypertens. 2016;34(7):1257-65.

21. Cho Y, Ann SH, Won K, Park G, Kim Y, Yang DH, et al. Association between insulin resistance, hyperglycemia, and coronary artery disease according to the presence of diabetes. Sci Rep-Uk. 2019:9(1):6129.

22. Lee EY, Yang HK, Lee J, Kang B, Yang Y, Lee S, et al. Triglyceride glucose index, a marker of insulin resistance, is associated with coronary artery stenosis in asymptomatic subjects with type 2 diabetes. Lipids Health Dis. 2016;15(1):155.

23. Alberti KG, Zimmet PZ. Definition, diagnosis and classification of diabetes mellitus and its complications. Part 1: diagnosis and classification of diabetes mellitus. Provisional report of a WHO Consultation. Diabetic Med. 1998;15(7):539-53.

24. American Diabetes Association. 2. Classification and diagnosis of diabetes: standards of medical care in diabetes-2020. Diabetes Care. 2020;43(Suppl 1):S14-31.

25. Roffi M, Patrono C, Collet J, Mueller C, Valgimigli M, Andreotti F, et al. 2015 ESC Guidelines for the management of acute coronary syndromes in patients presenting without persistent ST-segment elevation. Eur Heart J. 2016;37(3):267-315.

26. Levey AS, Coresh J, Greene T, Stevens LA, Zhang YL, Hendriksen S, et al. Using standardized serum creatinine values in the modification of diet in renal disease study equation for estimating glomerular filtration rate. Ann Intern Med. 2006;145(4):247-54.

27. Alfonso F, Byrne RA, Rivero F, Kastrati A. Current treatment of in-stent restenosis. J Am Coll Cardiol. 2014;63(24):2659-73.

28. Section of Interventional Cardiology of Chinese Society of Cardiology of Chinese Medical Association, Specialty Committee on Prevention and Treatment of Thrombosis of Chinese College of Cardiovascular Physicians, Editorial Board of Chinese Journal of Cardiology. Chinese guideline for percutaneous coronary intervention. Chin J Cardiol. 2016;44(5):382-400

29. Kozakova M, Morizzo C, Goncalves I, Natali A, Nilsson J, Palombo C. Cardiovascular organ damage in type 2 diabetes mellitus: the role of lipids and inflammation. Cardiovasc Diabetol. 2019:18(1):61.

30. Sharif S, Groenwold R, van der Graaf Y, Berkelmans G, Cramer MJ, Visseren $F$, et al. Mediation analysis of the relationship between type 2 diabetes and cardiovascular events and all-cause mortality: findings from the SMART cohort. Diabetes Obes Metab. 2019:21(8):1935-43.

31. Antuna-Puente B, Disse E, Rabasa-Lhoret R, Laville M, Capeau J, Bastard JP. How can we measure insulin sensitivity/resistance? Diabetes Metab. 2011:37(3):179-88.

32. Simental-Mendía LE, Rodríguez-Morán M, Guerrero-Romero F. The product of fasting glucose and triglycerides as surrogate for identifying insulin resistance in apparently healthy subjects. Metab Syndr Relat Disord. 2008:6(4):299-304.

33. Tuo $X$, Yuan J, Wang $X$, Xin Z. Identifying the insulin resistance index in nondiabetic Chinese subjects. Medicine. 2020;99(5):e19023.

34. Sánchez-García A, Rodríguez-Gutiérrez R, Mancillas-Adame L, GonzálezNava V, Díaz GA, Solis RC, et al. Diagnostic accuracy of the triglyceride and glucose index for insulin resistance: a systematic review. Int J Endocrinol. 2020;2020:4678526
35. Vasques AC, Novaes FS, de Oliveira MS, Souza JR, Yamanaka A, Pareja $J C$, et al. TyG index performs better than HOMA in a Brazilian population: a hyperglycemic clamp validated study. Diabetes Res Clin Pract. 2011;93(3):e98-100

36. Irace C, Carallo C, Scavelli FB, De Franceschi MS, Esposito T, Tripolino C, et al. Markers of insulin resistance and carotid atherosclerosis. A comparison of the homeostasis model assessment and triglyceride glucose index. Int J Clin Pract. 2013;67(7):665-72.

37. Navarro-González D, Sánchez-Iñigo L, Pastrana-Delgado J, FernándezMontero A, Martinez JA. Triglyceride-glucose index (TyG index) in comparison with fasting plasma glucose improved diabetes prediction in patients with normal fasting glucose: the Vascular-Metabolic CUN cohort. Prev Med. 2016;86:99-105.

38. Navarro-González D, Sánchez-Iñigo L, Fernández-Montero A, PastranaDelgado J, Martinez JA. TyG index change is more determinant for forecasting type 2 diabetes onset than weight gain. Medicine. 2016;95(19):e3646.

39. Su W, Chen S, Huang Y, Huang J, Wu P, Hsu W, et al. Comparison of the effects of fasting glucose, hemoglobin A1c, and triglyceride-glucose index on cardiovascular events in type 2 diabetes mellitus. Nutrients. 2019;11(11):2838

40. Park G, Cho Y, Won K, Yang YJ, Park S, Ann SH, et al. Triglyceride glucose index is a useful marker for predicting subclinical coronary artery disease in the absence of traditional risk factors. Lipids Health Dis. 2020;19(1):7.

41. Shi $W$, Xing $L$, Jing $L$, Tian $Y$, Yan $H$, Sun $Q$, et al. Value of triglycerideglucose index for the estimation of ischemic stroke risk: insights from a general population. Nutr Metab Cardiovasc Dis. 2020;30(2):245-53.

42. Jin JL, Cao YX, Wu LG, You XD, Guo YL, Wu NQ, et al. Triglyceride glucose index for predicting cardiovascular outcomes in patients with coronary artery disease. JThorac Dis. 2018;10(11):6137-46.

43. Jin J, Sun D, Cao Y, Guo Y, Wu N, Zhu C, et al. Triglyceride glucose and haemoglobin glycation index for predicting outcomes in diabetes patients with new-onset, stable coronary artery disease: a nested case-control study. Ann Med. 2018;50(7):576-86.

44. Mao Q, Zhou D, Li Y, Wang Y, Xu S, Zhao X. The triglyceride-glucose index predicts coronary artery disease severity and cardiovascular outcomes in patients with non-ST-segment elevation acute coronary syndrome. Dis Markers. 2019;2019:1-11.

45. Luo E, Wang D, Yan G, Qiao Y, Liu B, Hou J, et al. High triglyceride-glucose index is associated with poor prognosis in patients with acute STelevation myocardial infarction after percutaneous coronary intervention. Cardiovasc Diabetol. 2019;18(1):150.

46. Ma X, Dong L, Shao Q, Cheng Y, Lv S, Sun Y, et al. Triglyceride glucose index for predicting cardiovascular outcomes after percutaneous coronary intervention in patients with type 2 diabetes mellitus and acute coronary syndrome. Cardiovasc Diabetol. 2020;19(1):31.

47. Alizargar J, Bai C, Hsieh N, Wu SV. Use of the triglyceride-glucose index (TyG) in cardiovascular disease patients. Cardiovasc Diabetol. 2020;19(1):8

48. Alizargar J, Hsieh NC, Wu SV. Is the use of triglyceride-glucose (TyG) index to recognize glucose disorders really practical? Eur J Pediatr. 2020;179:1169 (Online ahead of print).

49. Zheng R, Mao Y. Triglyceride and glucose (TyG) index as a predictor of incident hypertension: a 9-year longitudinal population-based study. Lipids Health Dis. 2017;16(1):175.

50. Shi W, Liu S, Jing L, Tian Y, Xing L. Estimate of reduced glomerular filtration rate by triglyceride-glucose index: insights from a general Chinese population. Postgrad Med. 2019;131(4):287-94.

51. Low S, Khoo K, Irwan B, Sum CF, Subramaniam T, Lim SC, et al. The role of triglyceride glucose index in development of type 2 diabetes mellitus. Diabetes Res Clin Pract. 2018;143:43-9.

52. Ormazabal V, Nair S, Elfeky O, Aguayo C, Salomon C, Zuñiga FA. Association between insulin resistance and the development of cardiovascular disease. Cardiovasc Diabetol. 2018;17(1):122.

53. Laakso M, Kuusisto J. Insulin resistance and hyperglycaemia in cardiovascular disease development. Nat Rev Endocrinol. 2014;10(5):293-302.

54. Markus M, Rospleszcz S, Ittermann T, Baumeister SE, Schipf S, SiewertMarkus U, et al. Glucose and insulin levels are associated with arterial stiffness and concentric remodeling of the heart. Cardiovasc Diabetol. 2019;18(1):145 
55. Park K, Ahn CW, Lee SB, Kang S, Nam JS, Lee BK, et al. Elevated TyG index predicts progression of coronary artery calcification. Diabetes Care. 2019;42(8):1569-73.

56. Lee SB, Ahn CW, Lee BK, Kang S, Nam JS, You JH, et al. Association between triglyceride glucose index and arterial stiffness in Korean adults. Cardiovasc Diabetol. 2018;17(1):41

57. Zhao S, Yu S, Chi C, Fan X, Tang J, Ji H, et al. Association between macro- and microvascular damage and the triglyceride glucose index in community-dwelling elderly individuals: the Northern Shanghai Study. Cardiovasc Diabetol. 2019;18(1):95.

58. Nakagomi A, Sunami Y, Kawasaki Y, Fujisawa T, Kobayashi Y. Sex difference in the association between surrogate markers of insulin resistance and arterial stiffness. J Diabetes Complications. 2020;34(6):107442.

59. Liese AD, Roach AK, Sparks KC, Marquart L, D'Agostino RJ, Mayer-Davis EJ. Whole-grain intake and insulin sensitivity: the Insulin Resistance Atherosclerosis Study. Am J Clin Nutr. 2003;78(5):965-71.
60. Mazidi M, Katsiki N, Kengne AP, Mikhailidis DP, Banach M. Adiposity mediates the association between whole grain consumption, glucose homeostasis and insulin resistance: findings from the US NHANES. Lipids Health Dis. 2018;17(1):219.

61. Kelly SA, Hartley L, Loveman E, Colquitt JL, Jones HM, Al-Khudairy L, et al. Whole grain cereals for the primary or secondary prevention of cardiovascular disease. Cochrane Database Syst Rev. 2017;8(8):D5051.

\section{Publisher's Note}

Springer Nature remains neutral with regard to jurisdictional claims in published maps and institutional affiliations.
Ready to submit your research? Choose BMC and benefit from:

- fast, convenient online submission

- thorough peer review by experienced researchers in your field

- rapid publication on acceptance

- support for research data, including large and complex data types

- gold Open Access which fosters wider collaboration and increased citations

- maximum visibility for your research: over 100M website views per year

At BMC, research is always in progress.

Learn more biomedcentral.com/submissions 